\title{
Impfungen in der gynäkologischen Praxis
}

\author{
Michael Wojcinski
}

\begin{tabular}{|l|l|l|l|}
\hline Übersicht & & Grundsätzliche Hinweise & \\
\hline $\begin{array}{l}\text { Einleitung } \\
\text { Empfehlungen der STIKO }\end{array}$ & 4112 & zu Impfungen & 413 \\
\hline $\begin{array}{l}\text { Kategorien von Impfungen } \\
\text { Verantwortung des }\end{array}$ & 412 & $\begin{array}{l}\text { Die Standardimpfungen } \\
\text { Impfungen bei besonderen }\end{array}$ & 415 \\
\hline
\end{tabular}

\section{Einleitung}

Schutzimpfungen zählen zu den effektivsten und kostengünstigsten Präventivmaßnahmen der modernen Medizin. Keine andere medizinische Maßnahme hat so viel zur Gesunderhaltung des Menschen beigetragen wie die Schutzimpfungen.

Impfungen schützen die geimpfte Person (Individualschutz), aber auch Ungeimpfte durch die sogenannte Herdenimmunität. Bei einer Impfquote von 95\% der Bevölkerung werden auch die restlichen $5 \%$ Nichtgeimpfte geschützt - also die gesamte Bevölkerung (Populationsschutz).

\section{Vorteile des Impfens}

Impfungen reduzieren Erkrankungsfälle, verhindern Antibiotikaresistenzen, vermeiden Arbeitsunfähigkeiten, senken Behandlungskosten, verringern Klinikaufenthalte, verhindern Behinderungen und stellen somit die Grundlage für das Funktionieren einer Gesellschaft dar.

Der Frauenarzt ist der Impfarzt der Familie.

Die meisten Frauen sind gesund, wenn sie in unsere gynäkologischen Praxen kommen, sie sind offen für den Präventionsgedanken - sie kommen ja zum großen Teil zur „Vorsorge“. Die Frau entscheidet häufig in der
Familie über medizinische Fragen: Sie ist „der Gesundheitslotse“ der Familie. Spätestens bei Eintritt einer Schwangerschaft kommen der Partner und die Familie dazu und sind dann ebenfalls am Infektionsschutz interessiert.

In Deutschland existiert ein gut etabliertes Impfsystem, das gekennzeichnet ist durch jährlich aktualisierte, klare Empfehlungen der Ständigen Impfkommission (STIKO) und die Übernahme der Kosten durch die gesetzlichen und privaten Krankenkassen. Dennoch herrschen in der Bevölkerung Vorbehalte gegenüber Impfungen. Studien über den Impfstatus von Erwachsenen in Deutschland zeigen selbst bei den Standardimpfungen erhebliche Impfdefizite [1]. So verfügen $28,6 \%$ der Bevölkerung nicht über einen aktuellen Tetanusschutz, 42,9\% sind ohne Diphtherie-Auffrischung und 88,2\% ( +-West) bzw. 90,6\% ( $0^{\star}$-West) haben keine PertussisAuffrischung erhalten.

2012 ergab eine Umfrage der Bundeszentrale für gesundheitliche Aufklärung (BZGA), dass von den 4483 befragten Personen im Alter von 16-85 Jahren nur etwa 4-8\% als Impfgegner bezeichnet werden konnten, aber $31 \%$ den Impfungen ablehnend gegenüber standen und nur 37\% Schutzimpfungen klar befürworteten. Dies zeigte sich besonders bei der Bewertung der Masernimpfung: 28\% der Befragten hielten die Masernimpfung für unwichtig [2]. Das Resultat dieser Einstellung bewirkt, dass der von der Weltgesundheitsorganisation vorgegebene Zeitrahmen für die 
Elimination der Masern in Deutschland mehrfach verschoben werden musste, weil immer wieder regionale Masernhäufungen mit insgesamt weit über 2000 Fällen auftraten und somit das gesteckte Ziel von 1 Fall pro 1 Million Einwohner in weite Ferne geraten ließen.

Ein großer Anteil der Bevölkerung in Deutschland weist deutliche Impfdefizite auf - selbst bei den Standardimpfungen.

\section{Empfehlungen der STIKO}

Die Ständige Impfkommission (STIKO) am Robert KochInstitut in Berlin erarbeitet jährlich auf der Grundlage der aktuellen medizinischen und epidemiologischen Situation Impfempfehlungen, die als Voraussetzung für die Festsetzung öffentlicher Empfehlungen in den einzelnen Bundesländern dienen. Jeweils im August werden die neuen Empfehlungen im Epidemiologischen Bulletin Nr. 34 veröffentlicht. Erst wenn der Gemeinsame Bundesausschuss (GBA) diese Empfehlungen in die Schutzimpfungsrichtlinien übernommen hat und diese frühestens im Februar des Folgejahres im Bundesanzeiger abgedruckt worden sind, sind die neuen Empfehlungen Pflichtleistungen der gesetzlichen Krankenkassen. Die gegenwärtig gültigen Impfempfehlungen der STIKO wurden im August 2016 veröffentlicht [3].

Viele Experten sehen die uneinheitliche Entscheidung der einzelnen Bundesländer und die Verzögerung der Kostenübernahme neuer Impfempfehlungen als 2 von vielen Impfhindernissen in Deutschland an.

\section{Kategorien von Impfungen}

Die STIKO unterteilt die Impfungen in

- Standardimpfungen und

- Indikationsimpfungen.

\section{Standardimpfungen}

Bei Standardimpfungen handelt es sich um die Basisimpfungen, die jede Person routinemäßig erhalten sollte. Die Empfehlungen der STIKO stellen nach einem BGH-Urteil den Standard der Medizin dar [4]. Kehrt man diesen Gedanken um, dann ist die Unterlassung einer STIKO-empfohlenen Standardimpfung ein Ver- stoß gegen medizinisches Standardwissen, weshalb die Ablehnung einer Impfung gut dokumentiert werden sollte.

Die Standardimpfungen beginnen so früh wie immunologisch möglich bereits bei Säuglingen als Grundimmunisierungen und müssen teilweise lebenslang aufgefrischt werden.

\section{Tipp für die Praxis}

Frühgeborene erhalten unabhängig von ihrem Geburtsgewicht alle Impfungen, wie sie altersabhängig im STIKO-Impfplan vorgesehen sind.

\section{Indikationsimpfungen}

Dies sind Impfungen bei bestimmten Personengruppen mit höherer Gefährdung durch Infektionserkrankungen. Beispiele hierfür sind:

- Schwangere

- Personen mit chronischen Erkrankungen

- Personen mit Immundefekten

- vor immunsuppressiver Therapie

- vor Chemotherapie

- vor Bestrahlungen

- vor Operationen mit hohem Blutverlust

- vor Transplantationen

Aber auch Kontaktpersonen von Immundefizienten und alle Personen im Gesundheitsdienst gehören zu den Gruppen, die über die Standardimpfungen hinaus besonderen Schutzes vor weiteren impfpräventablen Erkrankungen bedürfen.

Zu den Indikationsimpfungen gehören auch Impfungen aufgrund eines erhöhten beruflichen Risikos sowie Reiseimpfungen, wobei die von der Weltgesundheits-

\section{Die Impfkategorien der STIKO}

Die einzelnen Impfkategorien sind in Tabelle $\mathbf{1}$ und 2 der STIKO-Empfehlungen mit folgenden Buchstaben gekennzeichnet:

$\begin{array}{ll}\mathrm{S} & =\text { Standardimpfung } \\ \mathrm{SM} & =\text { Standardimpfung für Mädchen } \\ \mathrm{G} & =\text { Grundimmunisierung } \\ \mathrm{A} & \quad=\text { Auffrischimpfung } \\ \mathrm{N} & =\text { Nachholimpfung } \\ \mathrm{I} & =\text { Nicht berufliche Indikationsimpfung } \\ \mathrm{B} & \text { = Berufliche Indikationsimpfung } \\ \mathrm{R} & \text { = Reiseimpfung }\end{array}$


organisation (WHO) veröffentlichten Informationen über die Gefahrensituation der einzelnen Reiseländer beachtet werden sollen.

Indikationsimpfungen gelten für bestimmte Personengruppen, beruflich Exponierte und Reisende.

\section{Auffrischimpfungen}

Auffrischimpfung werden erneute Impfungen gegen einen Krankheitserreger genannt, die nach früherer Grundimmunisierung wegen nachlassender Immunität in regelmäßigen Abständen erforderlich werden. Lebendimpfstoffe vermitteln meistens eine lebenslange Immunität, während eine Vielzahl von Totimpfstoffen nur einen zeitlich begrenzten Schutz bietet.

\section{Prophylaxe-Impfungen}

Postexpositionelle Prophylaxe bzw. andere Maßnahmen der spezifischen Prophylaxe (Tabelle 3 der STIKOEmpfehlungen) werden Maßnahmen genannt, die bei unzureichend geschützten Personen zu ergreifen sind, wenn Kontakt zu bestimmten Erregern bestand. Dazu gehören die postexpositionelle Impfung, die Gabe von Immunglobulinen oder eine Chemoprophylaxe sowie Riegelungsimpfungen, die in der Umgebung eines Erkrankten die Ausbreitung der Erkrankung verhindern sollen.

\section{Verantwortung des Frauenarztes}

Für den Frauenarzt beginnt mit dem ersten Besuch eines Mädchens im Alter von 9-17 Jahren in der gynäkologischen Praxis die Verantwortung, für den zukünftigen Impfschutz zu sorgen. Mit der HPV-Impfung werden zeitgleich der Schutz gegen Tetanus, Diphtherie, Pertussis und Poliomyelitis aufgefrischt und überprüft, ob die 3-malige Hepatitis-B-Impfung, die einmalige Impfung gegen Meningokokken $C$ sowie die 2-malige MMR-Impfung erfolgt sind und ein Schutz vor Varizellen besteht.

Keine Frau sollte ohne einen vollständigen Impfschutz in eine Schwangerschaft gehen.

Niemand sollte ungeimpft einer immunsupprimierenden Therapie unterzogen werden. Diskussionen um fehlende Impfungen in der Schwangerschaft oder unter immunsupprimierender Behandlung können vermieden werden, wenn jede Frau grundsätzlich über einen vollständigen Impfschutz verfügt, wobei besonders die Lebendimpfungen gegen Masern, Mumps und Röteln sowie Varizellen nicht fehlen dürfen.

\section{Grundsätzliche Hinweise zu Impfungen}

\section{Kontraindikationen}

Es gibt nur wenige Kontraindikationen für Impfungen. Eine akute schwere Erkrankung oder schwere Allergien gegen Impfstoffbestandteile (je nach Impfstoff z. B. Neomycin, Streptomycin, Hühnereiweiß) sind solche Kontraindikationen. Personen mit anaphylaktischer Reaktion nach oraler Hühnereiweißaufnahme dürfen keine Gelbfieber- oder Influenzaimpfstoffe erhalten. Eine Kontraindikation für Lebendimpfstoffe stellen Schwangerschaft, Immundefizienz und medikamentöse Immunsuppression dar.

Häufig werden Impfungen nicht durchgeführt, weil fälschlicherweise bestimmte Umstände als Kontraindikationen angesehen werden (s. Kasten).

Die einzige Ausnahme bei Impfungen in der Stillzeit stellt die Gelbfieberimpfung wegen vereinzelten Auftretens von Meningoenzephalitiden bei gestillten Säuglingen geimpfter Mütter dar.

\section{Falsche Kontraindikationen}

Keine Kontraindikationen = Impfungen erlaubt!

- banale Infekte, auch mit subfebrilen Temperaturen bis $38^{\circ} \mathrm{C}$

- Kontakt zu Personen mit ansteckenden Krankheiten

- Durchfallerkrankungen

- Neurodermitis u.a. Dermatosen

- chronische Erkrankungen

- Krampfanfälle in der Familie

- Stillen

- Antibiotika

- Fieberkrämpfe in der Anamnese

- niedrigdosierte Kortikosteroide (bis 20 mg/d Prednison-Äquivalent)

- Neugeborenenikterus

- Frühgeburten

- Multiple Sklerose

- Totimpfstoffe bei Immundefekten

- Schwangerschaft in der Umgebung des Impflings 
Einzig gegen Gelbfieber darf in der Stillzeit nicht geimpft werden.

\section{Impfabstände}

Für alle Impfungen gilt, dass jede Impfdosis zählt. Niemals müssen Impfungen einer begonnenen Grundimmunisierung wiederholt werden, wenn die Abstände der Impfungen in einer Impfserie nicht eingehalten wurden. Auch nach einer Unterbrechung von vielen Jahren erzeugt eine Auffrischimpfung wegen des immunologischen Gedächtnisses wieder einen sofort einsetzenden, lang anhaltenden Schutz.

Grundimmunisierungen sollten möglichst ohne Verzögerung nach Angaben in den Fachinformationen abgeschlossen werden. Angegebene Mindestabstände sollten dabei nicht unterschritten werden.

\section{Koadministration}

Grundsätzlich können alle notwendigen Impfungen mit Tot-, Toxoid- oder Lebendimpfstoffen zeitgleich verabreicht werden oder in zeitlich beliebiger Folge an mehreren Tagen. Können aber 2 Lebendimpfstoffe (z. B. MMR-Impfstoff und Varizellen-Impfstoff) nicht zeitgleich verabreicht werden, muss zwischen ihnen ein Mindestabstand von 4 Wochen liegen. Die durch die erste Lebendimpfung in Gang gesetzte Immunisierung würde sonst die Vermehrungsfähigkeit und damit die Immunogenität des zweiten Lebendimpfstoffs schwächen.

\section{Tipp für die Praxis}

Zwei Lebendimpfstoffe dürfen zeitgleich auch zusammen mit Totimpfstoffen verabreicht werden. Kann eine zeitgleiche Gabe von 2 Lebendimpfstoffen nicht durchgeführt werden, muss zwischen ihnen ein Mindestabstand von 4 Wochen eingehalten werden, um die Immunogenität der Impfstoffe nicht zu schwächen.

Einige Koadministrationen haben in Studien eine gegenseitige Abschwächung der Immunogenität verursacht, weshalb in den jeweiligen Fachinformationen der Impfstoffe entsprechende Hinweise aufgenommen wurden. So ist z. B. die Koadministration von Zostavax mit Pneumovax nicht empfohlen und die Koadministration von HPV-Impfstoffen bislang nur mit Tdap-IPV, Hepatitis A und B sowie tetravalentem Meningokokkenimpfstoff untersucht worden.

\section{Injektionsort}

Geimpft wird mit trockener, nicht entlüfteter Nadel in den M. deltoideus, 3 Querfinger von der Schulterhöhe entfernt, senkrecht zur Hautoberfläche. Alternativ ist der M. vastus lateralis geeignet.

Impfungen in den M. glutaeus maximus sind obsolet.

Sind mehrere Injektionen erforderlich, sollten sie auf beide Seiten verteilt werden. Zwischen 2 Impfungen auf einer Seite sollte ein Abstand von 2-3 cm eingehalten werden, um eine Vermischung der Impfstoffe im Muskel zu verhindern. Eine Aspiration vor der Impfung ist nicht erforderlich [3].

\section{Impfreaktionen}

Als „normale“ Impfreaktion kommt es gelegentlich an der Injektionsstelle zu Lokalreaktionen in Form von Rötung, Schwellung und Schmerz. Als Allgemeinreaktionen treten in einigen Fällen Fieber bis $39^{\circ} \mathrm{C}$, Müdigkeit, Kopfschmerzen oder grippale und gastrointestinale Beschwerden auf, die ohne Behandlung von alleine abklingen. Nach MMR-Impfungen können ein masernähnliches Exanthem oder Lymphknotenschwellungen, nach Windpockenimpfungen varizellenähnliche Bläschen auftreten, die aber keine Gefährdung darstellen.

\section{Tipp für die Praxis}

Eine namentliche Meldepflicht an das zuständige Gesundheitsamt besteht für Nebenwirkungen, die über die oben beschriebenen normalen Impfreaktionen hinausgehen.

\section{Formalia}

Dokumentation. Für alle Impfungen schreibt das Infektionsschutzgesetz (IfSG § 22) eine vollständige Dokumentation im Impfausweis oder in einer Impfbescheinigung vor. Diese muss folgende Angaben enthalten: Impfdatum, Chargen-Nummer und Handelsname des Impfstoffs sowie Name der Krankheit, gegen die geimpft wurde, und Stempel sowie Unterschrift des Arztes.

Aufklärung. Jede zu impfende Person ist nach dem Patientenrechtegesetz vom 20.02.2013 über die zu verhütende Krankheit und die geplanten Impfungen 
mündlich aufzuklären. Schriftliche Aufklärungsbögen können zur Ergänzung hilfreich sein. Ab zweiter Impfung im Rahmen der Grundimmunisierung oder bei Auffrischimpfungen ist keine besondere Aufklärung mehr erforderlich.

Impfeinwilligung. Die Einwilligung eines Elternteiles ist ausreichend. Minderjährige ab einem Alter von 14 Jahren können selbst wirksam in eine Behandlung einwilligen, wenn sie nach ihrer geistigen und sittlichen Reife die Bedeutung und Tragweite des Eingriffs ermessen können (Beurteilung durch den Arzt). Dies ist ab 16 Jahren im Allgemeinen gegeben. Bei Zweifel ist die Einwilligung durch den Sorgeberechtigten erforderlich. Für unbegleitete asylsuchende Kinder ist das Jugendamt sorgeberechtigt ( $§ 42$ SGB VIII).

Die zu impfende Person braucht nicht schriftlich in die Impfung einzuwilligen; die mündliche Impfeinwilligung ist üblich.

\section{Tipp für die Praxis}

Für den Arzt empfiehlt es sich, die Ablehnung einer empfohlenen Impfung gut zu dokumentieren, ggf. auch mit Unterschrift des Patienten.

\section{Impfstoff}

Impfstoffbezug. Hierzu sind die Bestimmungen der regionalen Kassenärztlichen Vereinigungen (KV) zu beachten. Die Verordnung von Impfstoffen erfolgt im Regelfall als Sprechstundenbedarf auf Kassenrezept zulasten des von der KV genannten Kostenträgers. Die Ziffern 8 und 9 sind auf dem Rezept einzutragen bzw. anzukreuzen. Im Feld Kassen-Nummer ist der Kostenträger anzugeben.

Da Impfstoffe ständig in der Praxis verfügbar sein sollten, stellt die namentliche Verordnung, die in manchen KV-Bereichen für z.B. HPV-Impfstoffe gefordert wird, eine zusätzliche Impferschwernis dar. Wirtschaftlichen Großpackungen und Kombinationsimpfstoffen soll der Vorzug gegeben werden, was bei Verordnung auf Einzelrezept zudem nicht einzuhalten ist.

Impfstofflagerung. Impfstoffe sind thermo- und lichtinstabil. Lebendimpfstoffe verlieren die Vermehrungsfähigkeit der attenuierten Viren bei Temperaturen $>8^{\circ} \mathrm{C}$.
Impfstoffe müssen im Kühlschrank bei $2-8^{\circ} \mathrm{C}$

lagern.

Im Kühlschrank sollten 2 Kontrollthermometer vorhanden sein. Impfstoffe dürfen nicht einfrieren und es sollte keine Lagerung in den Türfächern erfolgen. Lebendimpfstoffe müssen in Kühlkette transportiert werden. Eine verantwortliche Mitarbeiterin sollte die Lagerung überwachen.

\section{Delegation von Impfleistungen}

Mit Ausnahme der Indikationsstellung, der mündlichen Aufklärung und der Unterschrift im Impfausweis oder unter der Impfbescheinigung können alle anderen Impfleistungen einer Medizinischen Fachangestellten (MFA) übertragen werden. Die Gesamtverantwortung liegt beim Arzt, der sich auch bei Impfungen durch das Personal in Rufweite aufhalten muss.

\section{Kostenübernahme}

Mit Veröffentlichung der Schutzimpfungsrichtlinie des GBA sind die gesetzlichen Krankenkassen zur Übernahme der Standard- und nichtberuflichen Indikationsimpfungen (Kategorien S, SM und I) verpflichtet. Die beruflich veranlassten Indikationsimpfungen sind nicht im Leistungsumfang der Krankenkassen enthalten. Ebenso nicht die Reiseimpfungen, die aber bereits von verschiedenen Krankenkassen als Satzungsleistungen angeboten werden.

\section{Die Standardimpfungen}

Kinder erhalten in den ersten 2 Lebensjahren Standardimpfungen gegen Tetanus, Diphtherie, Pertussis, Haemophilus influenzae Typ b (Hib), Poliomyelitis, Hepatitis B, Pneumokokken, Rotaviren, Meningokokken C, Masern, Mumps, Röteln und Varizellen (Tab. 1). Vor der Einschulung werden Tetanus, Diphtherie und Pertussis (Tdap) erstmalig aufgefrischt (Tab. 2). Im Alter von 9-17 Jahren erhalten alle eine zweite Auffrischimpfung Tetanus, Diphtherie und Pertussis sowie die erste Poliomyelitis-Auffrischung (Tdap-IPV). Mädchen in diesem Alter erhalten zusätzlich die planmäßige Impfung gegen humane Papillomaviren (HPV). 


\begin{tabular}{|c|c|}
\hline Impfungen bis 23 Monate. & \\
\hline Kinder mit 2 Jahren sind geimpft gegen & $\begin{array}{l}\text { Anzahl der } \\
\text { Impfungen }\end{array}$ \\
\hline Tetanus & 4 \\
\hline Diphtherie & 4 \\
\hline Pertussis & 4 \\
\hline Hib & 4 \\
\hline Poliomyelitis & 4 \\
\hline Hepatitis B & 4 \\
\hline Pneumokokken & $3 / 4^{*}$ \\
\hline Rotaviren & $2 / 3$ \\
\hline Meningokokken C & 1 \\
\hline Masern, Mumps, Röteln & 2 \\
\hline Varizellen & 2 \\
\hline
\end{tabular}

\section{Tipp für die Praxis}

Bei jedem Patientenkontakt sollte darauf geachtet werden, dass die laut Impfkalender der STIKO vorgesehenen Impfungen auch erfolgt sind. Gegebenenfalls sollten alle fehlenden Impfungen bis zum Alter von 17 Jahren zulasten der Krankenkasse nachgeholt werden (mit Ausnahme der Rotavirus- und Pneumokokkenimpfung).

\section{Tetanus und Diphtherie}

\section{Epidemiologie}

Tetanus. Die Erkrankung ist weltweit verbreitet mit großen regionalen Unterschieden. Vor allem in feuchtwarmen Ländern mit niedrigen Impfraten und schlechter medizinischer Versorgung erkranken und sterben auch heute noch viele Menschen an dieser Krankheit.

In den Industriestaaten Europas ist die Tetanusinzidenz dank umfassender Impfungen sowie der verbesserten
Tabelle 2

Impfungen im Alter 5-6 Jahre = vor der Einschulung

Impfung gegen

Anzahl der

Impfungen

1. Auffrischimpfung von Tetanus, Diphtherie,

Pertussis $=$ Kombi-Impfstoff Tdap

Nachholimpfungen: Falls Kinder in den ersten Jahren nicht vollständig geimpft wurden, werden folgende Impfungen nachgeholt:

Poliomyelitis

Hepatitis B

Meningokokken C

Masern, Mumps, Röteln

Varizellen

2

Lebensbedingungen niedrig. In Deutschland wurden in den letzten Jahren unter 10 Erkrankungsfälle jährlich verzeichnet, überwiegend bei älteren weiblichen Erwachsenen. Die beobachtete Letalität betrug 25\% [5].

Diphtherie. Diphtherieerkrankungen treten in Deutschland nur noch in einzelnen Fällen einer Einschleppung auf. 2010 wurden in Europa noch 40 Fälle gemeldet. Bei sinkender Impfbereitschaft oder Verschlechterung der Hygienebedingungen könnten sowohl Tetanus- als auch Diphtheriefälle wieder vermehrt auftreten.

\section{Bei mangelnder Impfbereitschaft könnten die Erkrankungsraten für Tetanus und Diphtherie in} Deutschland wieder deutlich ansteigen.

\section{Impfstoffe}

Die Tetanus- und Diphtherie-Impfstoffe sind Toxoidimpfstoffe und enthalten inaktivierte Toxine. Die hoch immunogenen Impfstoffe werden als monovalente, meistens aber als Kombinationsimpfstoffe gegen Tetanus und Diphtherie eingesetzt.

Bereits 1998 hat die STIKO empfohlen, den monovalenten Tetanus-Impfstoff nicht mehr und ausschließlich Kombinationsimpfstoffe zu verwenden. 


\section{Impfempfehlung}

Die Tetanusimpfung ist eine für alle Personen bei fehlender oder unvollständiger Grundimmunisierung empfohlene Impfung. Sie wird immer in Kombination mit Diphtherie (Td-Impfstoff) durchgeführt. Nach STIKO sollen Frauen alle 10 Jahre eine gleichzeitige Auffrischung mit Pertussiskomponente erhalten (TdapImpfstoff). Für alle anderen gilt, dass zunächst nur die nächste fällige Tetanusauffrischung mit Tdap-Impfstoff, ggf. auch bei Notwendigkeit einer PoliomyelitisAuffrischung mit Tdap-IPV-Impfstoff erfolgen soll.

Es besteht für diese Impfung keine Altersbegrenzung nach oben.

\section{Grundimmunisierung und Auffrischung}

Da die STIKO die nächste fällige Auffrischung mit gleichzeitiger Pertussiskomponente (siehe Kap. Pertussis) empfiehlt, sollte bei einer erforderlichen Grundimmunisierung $-z$. B. bei nicht vorhandenen Impfdokumenten - die erste Impfdosis mit einem Kombinationsimpfstoff (Tdap) erfolgen. Diese wird durchgeführt nach dem Impfschema gemäß Tabelle 3.

\section{Schutzdauer}

Die Schutzdauer der Tetanus-Impfung beträgt 10 Jahre und länger. Regelmäßige Auffrischimpfungen bewirken einen 100\%igen Schutz. Der gewünschte Titer beträgt $>0,1 \mathrm{IE} / \mathrm{ml}$, als unterer Schutzgrenzwert gilt 0,01 IE $/ \mathrm{ml}$.

\section{Auffrischimpfung}

Die Tetanus-Auffrischimpfung erfolgt lebenslang alle 10 Jahre, wobei die STIKO empfiehlt, Erwachsene sollen die nächste fällige Td-Impfung einmalig als Tdap-, bei entsprechender Indikation Tdap-IPV-Kombinationsimpfung erhalten. Bei der Indikation zur Pertussisimpfung kann Tdap verwendet werden, auch wenn in einem Zeitraum von weniger als 5 Jahren zuvor ein TdImpfstoff geimpft worden ist [5]. Die STIKO hält dabei einen Abstand von 4 Wochen für ausreichend.

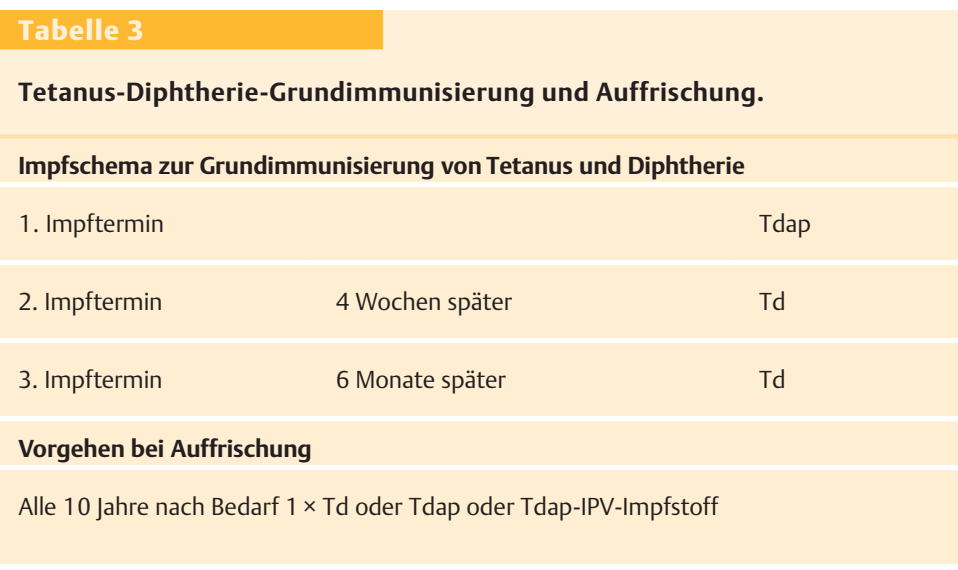

\begin{tabular}{|c|c|c|}
\hline \multicolumn{3}{|l|}{ Tabelle 4} \\
\hline \multicolumn{3}{|c|}{ Tetanus-Immunprophylaxe im Verletzungsfall. } \\
\hline $\begin{array}{l}\text { Erhaltene Anzahl der } \\
\text { Tetanus-Impfdosen }\end{array}$ & Tdap & Tetanus-Immunglobulin \\
\hline unbekannt & ja & ja \\
\hline $0-1$ & ja & ja \\
\hline 2 & ja & nein \\
\hline 3 oder mehr & nein & nein \\
\hline
\end{tabular}

\section{Passive Immunisierung}

Im Verletzungsfall und bei unsicherem Tetanusschutz wird humanes Tetanus-Immunglobulin postexpositionell simultan mit der aktiven Immunisierung nach den aktuellen STIKO-Empfehlungen verabreicht (Tab. 4).

Die STIKO unterscheidet zwar zur Vorgehensweise nach der Art der Verletzung („,saubere geringfügige Wunden/alle anderen Wunden“), was aber kritisch betrachtet werden sollte.

Auch bei geringfügigen Verletzungen kann es zu einer Tetanusinfektion kommen. 


\section{Pertussis}

\section{Epidemiologie}

Keuchhusten ist eine schwere und langwierige bakterielle Erkrankung, die weltweit verbreitet ist. In Deutschland werden jährlich ca. 100000 Erkrankungen beobachtet, wobei eine zunehmende Verlagerung in das Jugend- und Erwachsenenalter besteht. Eine prospektive Sentinel-Studie $($ KRESH-Studie $=$ Krefeld Rostocker Erwachsenen-Studie zur Hustengenese) an 1000 Patienten mit Husten über 7 Tagen Dauer konnte zeigen, dass jeder zehnte Patient unter Pertussis litt [6].

Bedrohlich sind die oft lebensgefährlichen Infektionen von noch ungeimpften Neugeborenen und jungen Säuglingen durch erkrankte Kontaktpersonen.

Die Immunität gegen Pertussis nach Impfung oder Erkrankung besteht etwa 8 bis höchstens 10 Jahre und nimmt dann wieder ab, sodass sich dadurch die Häufung der Erwachsenenerkrankungen erklärt.

\section{Impfstoffe}

Der heutige Impfstoff besteht nicht mehr aus ganzen Zellen, sondern entweder aus zellfreien Extrakten oder aus hochgereinigten einzelnen Komponenten (verschiedene Antigene) des Erregers. Zur Unterscheidung zum früher verfügbaren Ganzkeimimpfstoff kennzeichnet man in Abkürzungen den modernen azellulären Impfstoff mit einem vorangestellten „a“ (z. B. TdaP).

Pertussisimpfstoffe sind nicht mehr als monovalente Impfstoffe, sondern nur noch in Kombination mit Td oder Td-IPV als Dreifach- oder Vierfachimpfstoffe verfügbar.

\section{Tabelle 5}

Vorgehen bei nachzuholender Grundimmunisierung.

Impfschema zur Grundimmunisierung von Tetanus, Diphtherie, Pertussis und Poliomyelitis

\begin{tabular}{|c|c|c|c|}
\hline 1. Impftermin & & Tdap-IPV & \\
\hline 2. Impftermin & 4 Wochen später & 2. $\mathrm{Td}$ & 2. IPV \\
\hline 3. Impftermin & 5 Monate später & 3. $\mathrm{Td}$ & 3. IPV \\
\hline Auffrischimpfung & alle 10 Jahre & Tdap-IPV & \\
\hline
\end{tabular}

\section{Impfempfehlung}

Jede notwendige Tetanusauffrischung soll einmalig mit einem Kombinationsimpfstoff erfolgen, der gleichzeitig auch vor Pertussis schützt. Alle Frauen im gebärfähigen Alter sollten alle 10 Jahre eine Auffrischimpfung gegen Tetanus-Diphtherie-Pertussis, ggf. auch IPV erhalten.

Alle engen Haushaltskontaktpersonen von Neugeborenen sollten möglichst bis 4 Wochen vor der Geburt des Kindes gegen Pertussis geimpft werden.

Eine fehlende Pertussisimpfung der Mutter ist in den ersten Tagen post partum nachzuholen.

Die STIKO empfiehlt noch nicht die in anderen Ländern bereits empfohlene Impfung in der 27.-36. SSW, wodurch den Neugeborenen für die ersten Lebenswochen sogar Leihantikörper mitgegeben werden könnten, wohingegen Pertussis sonst weder nach Erkrankung noch nach Impfung vor dieser Zeit einen Nestschutz hinterlässt $[7,8]$.

Für Personal im Gesundheitsdienst sowie in Gemeinschaftseinrichtungen besteht eine arbeitsmedizinische Indikation zur Pertussisimpfung.

\section{Tipp für die Praxis}

Alle Schwangeren sollten frühzeitig auf den Impfschutz in der Familie und im Umgebungsbereich des Neugeborenen hingewiesen werden. Potenziellen Kontaktpersonen sollte die Möglichkeit der Impfausweiskontrolle bei Frauenärzten und die erforderliche Impfung angeboten werden.

Besonders wichtig bei Kontaktpersonen ist der Schutz gegen Influenza, Masern und Pertussis.

\section{Grundimmunisierung}

Erwachsene erhalten eine einzelne Impfung mit einem Kombinationsimpfstoff (Tdap, Tdap-IPV).

Obwohl die Fachinformation zu den Kombinationsimpfstoffen den Hinweis enthält, dass diese Impfstoffe nur zur Auffrischung zugelassen sind, können diese auch im Rahmen einer notwendigen Grundimmunisierung, z. B. bei nicht vorhandenen Impfdokumentationen eingesetzt werden (s. Tab. 5) [9]. 


\section{Schutzdauer}

Die Schutzdauer der Tdap-/Tdap-IPV-Impfung beträgt 10 Jahre. Ein akzeptierter Grenzwert für eine positive Impfantwort existiert bez. Pertussis nicht.

\section{Auffrischimpfung}

Im Rahmen der frauenärztlichen Impfstrategie ist darauf zu achten, dass alle Kinder und Jugendlichen bis zum 18. Lebensjahr über einen Basisimpfschutz gegen Pertussis verfügen und lückenlos zwischen dem 9. und 17. Lebensjahr die Auffrischimpfung erhalten haben.

Es wird empfohlen, alle 10 Jahre erneut gegen Pertussis mit Kombinationsimpfstoffen zu impfen.

Erwachsene sollen die nächste fällige Tetanusimpfung einmalig als Tdap, bei entsprechender Indikation TdapIPV-Kombinationsimpfung erhalten. Bei der Indikation zur Pertussisimpfung kann Tdap auch nach kurz zuvor durchgeführter Td-Impfung verwendet werden, wenn ein Mindestabstand von einem Monat eingehalten wird.

Passive Immunisierung ist bei Pertussis nicht möglich.

\section{Poliomyelitis}

\section{Epidemiologie}

Vor Einführung der Impfung gegen Poliomyelitis war diese Krankheit eine ständige Bedrohung der Weltbevölkerung. Erst mit Einführung der Impfung Ende der 50er-Jahre verlor die Erkrankung ihren Schrecken. Es kam zur rasanten Abnahme der Häufigkeit der Erkrankung, und 2002 wurde Europa nach Amerika und Australien von der WHO als poliofrei zertifiziert. 2014 errang auch Südostasien diesen Status. Heute kommt Polio nur noch in 3 Ländern der Welt endemisch vor: Afghanistan, Pakistan und Nigeria.

Von den 3 Polio-Wildvirustypen sind bereits die Typen II (1999) und III (2012) weltweit eradiziert.

Bei Einstellungen der Impfaktivitäten in Krisen- und Katastrophengebieten kommt es aber immer wieder schnell zum Ausbreiten der Erkrankung in andere Länder, wie zuletzt 2012 am Horn von Afrika, in Somalia und in Syrien mit dem Virustyp I geschehen. Das RKI verfügte damals, auch in Deutschland großzügig wieder gegen Poliomyelitis zu impfen, um Einschleppungen nach Deutschland zu verhindern.

\section{Tipp für die Praxis}

Die Polioimpfung ist die einzige Impfung, die auch als Reiseimpfung von allen Krankenkassen bezahlt wird, um Einschleppung von Poliowildviren in das poliofreie Deutschland zu verhindern.

Impfstoffe

Der Poliomyelitis-Einzelimpfstoff (IPV) und die Kombinationsimpfstoffe (Td-IPV, Tdap-IPV) enthalten inaktivierte Polioviren der Typen I, II, III. Die früher verwendeten oralen Lebendimpfstoffe (OPV) sind in Deutschland nicht mehr verfügbar.

\section{Impfempfehlung}

Alle Neugeborenen erhalten in den ersten beiden Lebensjahren 4 Polioimpfungen als Grundimmunisierung, Jugendliche zwischen 9 und 17 Jahren eine Auffrischung. Alle Erwachsenen sollten somit über eine vollständige Grundimmunisierung und wenigstens eine Auffrischimpfung nach 10 Jahren verfügen.

Eine arbeitsmedizinische Indikation besteht für medizinisches Personal, das engen Kontakt zu Erkrankten haben kann, sowie für Laborpersonal mit Poliorisiko.

Eine weitere Indikation besteht für Reisende in Regionen mit Infektionsrisiko sowie aus Risikogebieten einreisende Aussiedler, Flüchtlinge und Asylbewerber.

\section{Grundimmunisierung}

Eine komplette Poliomyelitis-Immunisierung umfasst 2 bzw. 3 Impfungen (je nach Impfstoff, Herstellerangaben beachten) plus 1 Auffrischimpfung 10 Jahre nach der Grundimmunisierung (s.Tab. 6).

\section{Schutzdauer}

Die Schutzwirkung tritt 2 Wochen nach der 2. Impfung ein und hält mindestens 10 Jahre nach kompletter Grundimmunisierung an. 


\section{Tabelle 6}

Grundimmunisierung mit IPV-Impfstoff (je nach Impfstoff mit

2 oder 3 Impfdosen).

Impfschema zur Grundimmunisierung Poliomyelitis

\begin{tabular}{|c|c|c|}
\hline 1. Impftermin & & 1. IPV \\
\hline (2. Impftermin) & 4 Wochen später & 2. IPV* \\
\hline 2./3.Impftermin & 6 Monate später & 3. IPV \\
\hline
\end{tabular}

\section{Auffrischimpfung}

Sie wird bei allen Personen ohne einmalige Auffrischimpfung durchgeführt; eine routinemäßige Auffrischung bei Jugendlichen wird nach dem vollendeten 18. Lebensjahr nicht empfohlen.

Bei Reisen in Gefährdungsgebiete und länger als 10 Jahre zurückliegender Impfung ist eine Auffrischimpfung zulasten der Krankenkasse erforderlich.

\section{Passive Immunisierung}

Bei Nichtgeimpften können im Kontaktfall humane Standardimmunglobuline mindestens $0,2-0,5 \mathrm{ml} / \mathrm{kg}$ KG in den ersten 5 Tagen nach Exposition als Versuch appliziert werden.

\section{Hepatitis B}

\section{Epidemiologie}

Das Hepatitis-B-Virus wird durch Geschlechtsverkehr und Blutkontakte, durch kontaminierte Kanülen (intravenöser Drogenmissbrauch), Nadelstichverletzung, Piercing, Tätowieren etc. übertragen. Ca. 5\% der Gesamtbevölkerung zeigen in Deutschland eine abgelaufene Infektion (Anti-HBc-positiv/HBsAg-negativ) und ca. 0,3\% eine chronische Infektion (HBsAG-positiv) [10].

Die Hepatitis B verläuft in 60-70\% der Fälle asymptomatisch, die Chronifizierung sinkt mit steigendem Alter. Bei Säuglingen beträgt sie etwa $90 \%$, bei Jugendlichen und Erwachsenen 5-10\%. Als Spätfolgen sind Leberzirrhose und Leberzellkarzinom bekannt [11,12]. Eine vertikale Transmisssion ist bei hoher Viruslast in

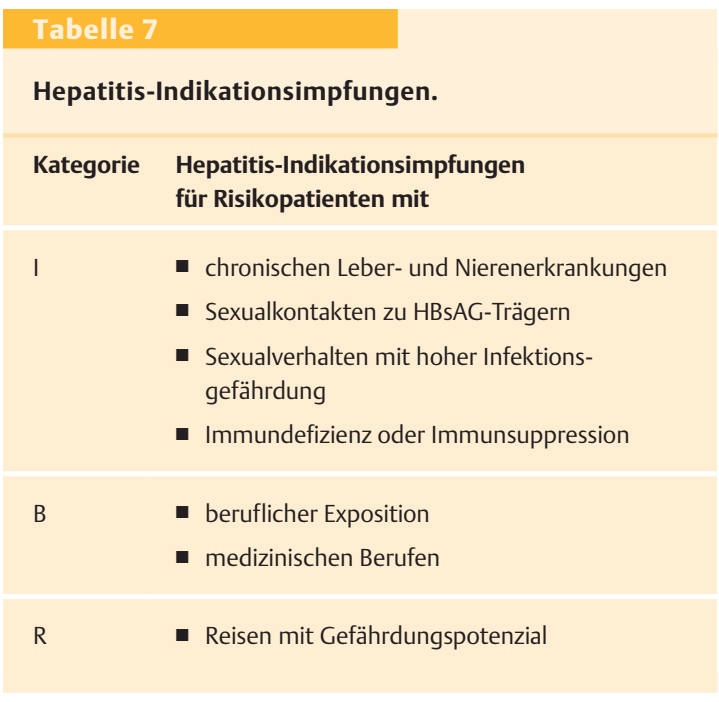

der Schwangerschaft und perinatal durch maternofetale Transfusion sowie kurz nach der Geburt durch engen Kontakt zu infektiösen Personen möglich.

\section{Impfstoffe}

Bei den Impfstoffen handelt es sich um gentechnologisch hergestelltes Oberflächenantigen des HepatitisB-Virus. Es steht für Erwachsene neben monovalenten Impfstoffen ein Kombinationsimpfstoff von Hepatitis B und Hepatitis A zur Verfügung.

\section{Impfempfehlung}

Impfung aller Kinder und Jugendlichen von 0-17 Jahren, Impfung von Risikogruppen bei Erwachsenen (Indikationsimpfung) (s. Tab. 7).

\section{Tipp für die Praxis}

Entsprechend den Mutterschaftsrichtlinien ist bei allen Schwangeren nach der 32. SSW möglichst nahe am Geburtstermin das Blutserum auf HBs-Antigen zu untersuchen. Ist das Ergebnis positiv, erhält das Neugeborene innerhalb von 12 Stunden post partum eine Simultanprophylaxe durch aktive und passive Immunisierung.

\section{Grundimmunisierung}

Die Grundimmunisierung von Kindern und Jugendlichen bis zum 18. Lebensjahr erfolgt nach dem Standardschema: 0-1-6 (12) Monate. Eine Kontrolle des 
Impferfolgs auf Anti-HBs ist nur für bestimmte Indikationen erforderlich (siehe Kasten der STIKO-Empfehlungen) [3].

\section{Schutzdauer}

Nach 3-maliger Impfung ist ein lebenslanger Schutz anzunehmen. Der lebenslange Schutz kann durch den einmaligen Nachweis von > 100 IE/l Anti-HBs dokumentiert werden, was z. B. im Bereich der Arbeitsmedizin gefordert wird.

\section{Auffrischimpfung}

Nach kompletter Grundimmunisierung wird derzeit keine generelle Auffrischimpfung mehr empfohlen.

\section{Passive Immunisierung}

Eine passive Immunisierung ist indiziert für Neugeborene HBsAg-positiver Mütter (Tab. 7) oder nach Hepatitis-B-Kontakt einer ungeschützten Person (Tab. 8).

\section{Postexpositionelle Hepatitis-B-Prophylaxe}

Je nach Höhe des Anti-HBs-Wertes erfolgt die Hepatitis-B-Prophylaxe gemäß dem in Tabelle 9 aufgeführten Schema.

\section{Impfschutz}

Nach heutiger Erkenntnis braucht nach einer vollständigen Grundimmunisierung keine Auffrischimpfung mehr zu erfolgen. Im arbeitsmedizinischen Bereich gilt der einmalige Nachweis von > 100 IE/l Anti-HBs als lebenslanger Schutz.

\section{Masern - Mumps - Röteln (MMR)}

\section{Epidemiologie}

Masern, Mumps und Röteln (MMR) sind hochkontagiöse Viruserkrankungen, die nicht nur bei Kindern, sondern zunehmend auch bei Erwachsenen vorkommen.

\begin{tabular}{|c|c|}
\hline Tabelle 8 & \\
\hline Vorgehen bei & renen abhängig vom HBsAg-Befund der Mutter. \\
\hline HBsAg & Konsequenz \\
\hline positiv & $\begin{array}{l}\text { innerhalb von } 12 \text { Stunden aktive und passive Immunisierung } \\
\text { (1. Hep-B-Kinderimpfdosis und } 1 \mathrm{ml} \mathrm{Hep-B-Immunglobulin)}\end{array}$ \\
\hline negativ & $\begin{array}{l}\text { nichts erforderlich } \\
\text { (Impfung des Neugeborenen gemäß Impfplan) }\end{array}$ \\
\hline unbekannt & umgehend HBsAg-Bestimmung \\
\hline $\begin{array}{l}\text { nicht innerhalb } \\
\text { von } 12 \text { Stunden } \\
\text { zu bestimmen }\end{array}$ & 1. Dosis Hepatitis-B-Impfstoff \\
\hline
\end{tabular}

\section{Tabelle 9}

\begin{tabular}{|c|c|c|}
\hline aktueller Anti-HBs-Wert & $\begin{array}{l}\text { erforderliche Gabe } \\
\text { von Hep-B-Impfstoff }\end{array}$ & $\begin{array}{l}\text { erforderliche Gabe von } \\
\text { Hep-B-Immunglobulin }\end{array}$ \\
\hline$>100 \mathrm{IE} / \mathrm{l}$ & nein & nein \\
\hline $10-99 \mathrm{IE} / \mathrm{I}$ & ja & nein \\
\hline$<10 \mathrm{IE} / \mathrm{I}$ & ja & ja \\
\hline $\begin{array}{l}\text { nicht innerhalb } 48 \mathrm{~h} \\
\text { zu bestimmen }\end{array}$ & ja & ja \\
\hline
\end{tabular}

Masern. Masern sollten schon längst ausgerottet sein, aber impfkritische Eltern verweigern immer wieder aus nicht nachvollziehbaren Gründen ihren Kindern den nötigen Impfschutz. In den letzten Jahren traten in Deutschland immer noch bis zu 2500 Masernfälle auf und jährlich sterben noch Kinder an den Folgen der Erkrankung. Eine der gefährlichsten Folgen ist die noch Jahre nach der Masernerkrankung auftretende subakute sklerosierende Panenzephalitis (SSPE), deren Letalität 100\% nach einem Krankheitsverlauf von 1 -3 Jahren beträgt. In den Jahren 2003-2009 waren 31 Kinder davon betroffen. Das Risiko für eine SSPE wird bei Masern bei unter 5-Jährigen auf $1: 1700$ bis $1: 3300$ Fälle geschätzt. Je jünger das Kind bei der Maserninfektion, umso höher das Risiko für eine SSPE [13].

Bei Masern in der Schwangerschaft besteht keine signifikant erhöhte Abortrate oder Gefahr für konnatale Defekte. 
Mumps. Die Mumpserkrankung zeigt ebenfalls keine neonatalen Defekte nach Erkrankung in der Schwangerschaft. Die jährliche Zahl der Meldungen liegt in Deutschland bei 800 Fällen. An Komplikationen treten in $60 \%$ der Fälle ZNS-Beteiligungen auf, wobei $1 \%$ Enzephalitiden mit einer Letalität von 1,5\% betreffen. Taubheit, Orchitis und Oophoritis sowie Mastitiden können Folge einer Mumpserkrankung sein. Eine nach Orchitis auftretende Sterilität ist gegenüber früheren Annahmen eher selten [14].

Röteln. Röteln treten nur noch vereinzelt auf und haben dank konsequenter Serokontrollen und Impfungen in den letzten Jahren keine Fälle von Embryopathien mehr verursacht. Weiterhin muss zum Erhalt dieser Sicherheit konsequent gegen Röteln geimpft werden.

\section{Impfstoffe}

Monovalente Impfstoffe haben keine Indikation mehr. Der Kombinationsimpfstoff gegen Masern, Mumps und gleichzeitig Röteln (MMR-Impfstoff) ist ein Lebendimpfstoff, der auch dann verwendet werden soll und kann, wenn gegen die eine oder andere Erkrankung schon Immunität besteht.

\section{Impfempfehlung}

Erst ab dem Alter von 11 Monaten kann die MMRImpfung durchgeführt werden, weil vorher vorhandene Leihantikörper der Mutter den Impferfolg verhindern könnten.

Wurde die 1. Impfung vor dem vollendeten 11. Lebensmonat durchgeführt, muss die 2. Impfung bereits zu Beginn des 2. Lebensjahrs erfolgen. Frühestens 4 Wochen nach der 1. Impfung kann die 2. MMR-Impfung gegeben werden, spätestens soll sie zwischen dem 15. und 23. Lebensmonat erfolgen (s. Tab. 10).

Nicht in diesem frühen Lebensalter verabreichte Impfungen sollen bis zum 18. Geburtstag nachgeholt werden. Ab 18 Jahre sollten alle Personen, die nach 1970 geboren sind und nicht oder nur einmal gegen Masern geimpft wurden, noch eine MMR-Impfung erhalten. Die Indikationen sind in Tabelle $\mathbf{1 1}$ zusammengefasst.

Gebärfähige Frauen müssen zum Nachweis der Immunität über 2 dokumentierte Rötelnimpfungen verfügen.

\begin{tabular}{|c|c|c|c|}
\hline \multicolumn{4}{|c|}{ MMR-Impfempfehlungen. } \\
\hline Wer? & Alter & Bedingung & Erforderliche Impfung \\
\hline Kinder und Jugendliche & 1-17 Jahre & keine & $2 \times M M R$ \\
\hline Erwachsene & nach 1970 Geborene & nicht oder nur einmal geimpft & $1 \times \mathrm{MMR}$ \\
\hline \multirow[t]{2}{*}{ Frauen } & im gebärfähigen Alter & ungeimpft & $2 \times M M R$ \\
\hline & & $1 \times$ Röteln & $1 \times \mathrm{MMR}$ \\
\hline \multirow[t]{5}{*}{ Säuglinge und Kleinkinder } & ab 9 Monate & vor Aufnahme in & 1. MMR sofort \\
\hline & & $\begin{array}{l}\text { Gemeinschaftseinrichtung, } \\
\text { nach Masernkontakt }\end{array}$ & $\begin{array}{l}\text { 2. MMR schon zu Beginn } \\
\text { des 2. Lebensjahrs }\end{array}$ \\
\hline & 6-8 Monate & individuelle Abwägung & 1. MMR sofort \\
\hline & & & 2. MMR und \\
\hline & & & 3. MMR im 2. Lebensjahı \\
\hline
\end{tabular}




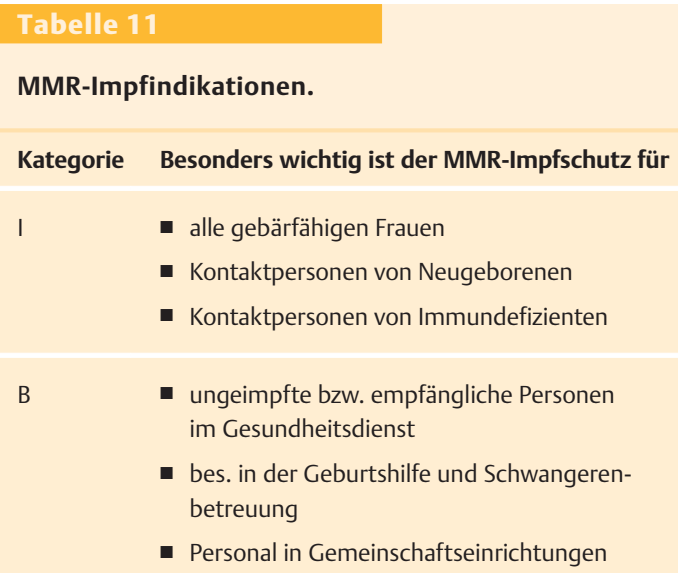

- bes. in der Geburtshilfe und Schwangerenbetreuung

- Personal in Gemeinschaftseinrichtungen

\section{Tipp für die Praxis}

Lebendimpfstoffe (MMR-Impfstoff und Varizellenimpfstoff) dürfen bei bekannter Schwangerschaft nicht angewendet werden. Versehentliche Impfungen in der Schwangerschaft haben aber nur ein theoretisches Risiko und weltweit noch niemals einen Schaden verursacht. Sie wären niemals ein Grund für einen Schwangerschaftsabbruch.

Vor keiner Impfung muss ein Schwangerschaftstest gemacht werden.

\section{Grundimmunisierung}

Frühestens 4 Wochen nach erster Impfdosis wird eine Komplettierung der Impfung mit einer zweiten MMR-Impfstoffdosis durchgeführt. Ist bereits eine MMR-Impfung dokumentiert, wird eine zweite MMR-Impfdosis appliziert. Bei Mädchen und Frauen ist keine Röteln-Antikörper-Kontrolle mehr erforderlich, wenn 2 Rötelnimpfungen dokumentiert sind.

Auch bei anamnestisch angegebener Masern-, Mumpsoder Rötelnerkrankung sollten die 2 MMR-Impfungen durchgeführt werden.

\section{Schutzdauer}

Nach 2 dokumentierten MMR-Impfungen kann von lebenslangem Schutz ausgegangen werden. Auch bei grenzwertigen oder negativen IgG-Werten sind bei 2 dokumentierten MMR-Impfungen keine weiteren Maßnahmen erforderlich [15].

\section{Auffrischimpfungen}

Nach 2 dokumentierten MMR-Impfungen sind keine weiteren Impfungen erforderlich.

\section{Tipp für die Praxis}

Nachweis von 2 dokumentierten Rötelnimpfungen bedeutet Rötelnimmunität. Bestimmung des RötelnIgG ist nur noch bei Schwangeren ohne 2 dokumentierte Rötelnimpfungen erforderlich.

Da kein Röteln-Hyperimmunglobulin mehr zur Postexpositionsprophylaxe zur Verfügung steht, ist es besonders wichtig, darauf zu achten, dass jede gebärfähige Frau über 2 dokumentierte Rötelnimpfungen verfügt.

\section{Postexpositionsprophylaxe}

Personen mit unklarem Masern- oder Mumpsschutz, ohne Impfung oder mit nur einer dokumentierten Impfung in der Kindheit sollten möglichst innerhalb von 3 Tagen nach Masernkontakt mit dem MMR-Impfstoff geimpft werden. Ungeimpfte erhalten 2 Impfdosen im Abstand von 4 Wochen, bisher einmal Geimpfte erhalten eine Impfung. Immundefiziente Personen ohne Masernschutz können nach Masernkontakten eine passive Immunisierung mit Standardimmunglobulinen erhalten.

\section{Tipp für die Praxis}

Die Anwendung von Lebendimpfstoffen ist bei Immundefizienten oder bei therapeutischer Immunsuppression kontraindiziert.

Bei versehentlicher Impfung sind sofort geeignete Maßnahmen zur Inaktivierung der Impfung durch Immunglobulingabe und antivirale Therapie einzuleiten.

\section{Varizellen}

\section{Epidemiologie}

Vor der Einführung der Varizellen-Impfung kam es in Deutschland zu jährlich etwa 750000 Erkrankungen. Damit stellten die Windpocken die häufigste Erkrankung im Kindesalter dar.

Etwa 96\% aller gebärfähigen Frauen verfügen über Varizellen-Antikörper und sind somit vor einer erneuten Erkrankung geschützt [15]. Jährlich werden in den letzten Jahren bis zu 20000 Erkrankungsfälle gemeldet. 
Diaplazentar kann es in 1-2\% der Windpocken von Schwangeren zur Übertragung auf den Feten und zum fetalen Varizellensyndrom kommen, sofern die Erkrankung zwischen der 5. und 21. Schwangerschaftswoche, in Einzelfällen auch bis zur 24. SSW auftritt.

Besonders bedrohlich sind Windpocken 5 Tage vor bis 2 Tage nach Geburt.

\section{Tipp für die Praxis}

Alle gebärfähigen Frauen müssen vor einer Varizelleninfektion geschützt sein. Dazu gehört, dass bei jeder gebärfähigen Frau gemäß der Richtlinie des Gemeinsamen Bundesausschusses zur Empfängnisregelung und zum Schwangerschaftsabbruch, Stand 15. 09.2011 (ESA-Richtlinie), bereits vor einer Schwangerschaft der Varizellen-Immunstatus kontrolliert und bei Seronegativität eine Impfung gegen Varizellen durchgeführt wird [16]. Für die Blutabnahme ist die Abrechnungsnummer EBM 01828 anzusetzen.

\section{Impfstoff}

Es handelt sich um einen monovalenten Lebendimpfstoff aus attenuierten, vermehrungsfähigen VarizellaZoster-Viren. Daneben gibt es einen Vierfach-Impfstoff gegen Masern, Mumps, Röteln und Varizellen, der nur für Kinder zwischen 9 Monaten und 12 Jahren zugelassen ist und der nur als Zweitimpfung nach primär erfolgter getrennter MMR- und Varizellenimpfung angewandt werden soll.

\section{Impfempfehlung}

Erst ab dem Alter von 11 Monaten kann die Varizellenimpfung durchgeführt werden, weil vorher vorhandene Leihantikörper der Mutter den Impferfolg verhindern könnten.

Frühestens 4 Wochen nach der 1. Impfung kann die 2. Varizellenimpfung gegeben werden, spätestens soll sie zwischen dem 15. und 23. Lebensmonat erfolgen.

Nicht in diesem frühen Lebensalter verabreichte Impfungen sollen bis zum 18. Geburtstag nachgeholt werden. Jede gebärfähige Frau sollte auf vorhandene Varizellen-Antikörper zum Nachweis des Schutzes untersucht werden.

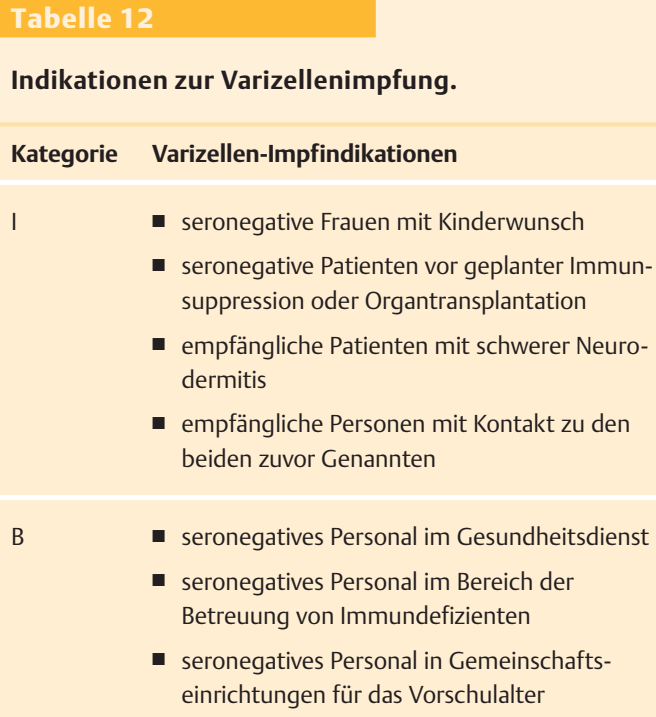

- empfängliche Personen mit Kontakt zu den beiden zuvor Genannten

B

- seronegatives Personal im Gesundheitsdienst

- seronegatives Personal im Bereich der Betreuung von Immundefizienten

- seronegatives Personal in Gemeinschaftseinrichtungen für das Vorschulalter

Seronegative Frauen müssen 2-mal im Mindestabstand von 4 Wochen eine Varizellenimpfung erhalten.

Die Varizellen-Impfempfehlungen sind in Tabelle 12 zusammengefasst.

\section{Grundimmunisierung}

Frühestens 4 Wochen nach der ersten Impfdosis wird eine Komplettierung der Impfung mit einer zweiten Varizellen-Impfstoffdosis durchgeführt. Ist bereits eine Varizellenimpfung dokumentiert, wird eine zweite Varizellenimpfdosis appliziert. Bei Mädchen und Frauen ist nach den ESA-Richtlinien eine Kontrolle der Varizellen-Antikörper vorgeschrieben, auch wenn 2 Varizellenimpfungen dokumentiert sind.

\section{Tipp für die Praxis}

Auch bei Varizellen zählen nur die dokumentierten Impfungen, weil man sich nie zweifelsfrei auf anamnestische Angaben zu Impfungen verlassen kann.

\section{Schutzdauer}

Nach 2 dokumentierten Varizellenimpfungen kann von lebenslangem Schutz ausgegangen werden. 


\section{Auffrischimpfungen}

Nach 2 dokumentierten Varizellenimpfungen sind keine weiteren Impfungen erforderlich.

\section{Postexpositionsprophylaxe}

Personen mit unklarem Varizellenschutz und ohne Impfung sollten möglichst innerhalb von 5 Tagen nach Varizellenkontakt geimpft werden. Ungeimpfte erhalten 2 Impfdosen im Abstand von 4-8 Wochen.

Dies gilt nicht für Personengruppen, die keine Lebendimpfstoffe erhalten dürfen: Schwangere oder immundefiziente Personen ohne Varizellenschutz können nach Varizellenkontakt eine passive Immunisierung mit VZV-Immunglobulin erhalten. Diese kann ggf. mit einer antiviralen Chemotherapie kombiniert werden.

\section{Herpes Zoster}

Herpes Zoster entsteht durch Reaktivierung von lebenslang in den Spinal- und Hirnnervenganglien ruhenden VZ-Wildviren. Herpes Zoster tritt vorwiegend bei immungeschwächten und älteren Personen nach dem 50. Lebensjahr auf, kann aber auch jüngere gesunde Personen treffen.

Ein Herpes Zoster der Schwangeren ist ohne Konsequenzen für den Embryo oder Feten.

\section{Impfstoff}

Seit einigen Jahren wird ein Zosterimpfstoff in den USA angewandt, der jetzt auch in Deutschland zur Verfügung steht. Es ist ein 14-fach höher dosierter Varizellen-Lebendimpfstoff, der ab dem Alter von 50 Jahren zugelassen ist. Es handelt sich bei der Zosterimpfung um eine Privatleistung. Besonders gute Erfolge zeigt dieser Impfstoff zur Verhinderung einer postherpetischen Neuralgie. Als Lebendimpfstoff ist er kontraindiziert bei den besonders gefährdeten Personen mit Immunschwäche, und er zeigt eine abnehmende Schutzwirkung in höherem Alter. Ein Totimpfstoff wird in Zukunft erwartet.

\section{Humane Papillomaviren (HPV)}

\author{
Epidemiologie
}

Von den bisher bekannten über 100 Typen des HPVirus ist eine Infektion mit den Typen 16 und 18 für ca. $72 \%$ aller entstandenen Zervixkarzinome in Europa verantwortlich [17]. Weitere gut $16 \%$ der Zervixkarzinome werden durch die HPV-Typen 31, 33, 45, 52 und 58 ausgelöst, die im nonavalenten HPV-Impfstoff zusätzlich zu den Hochrisikotypen 16 und 18 enthalten sind [18].

Laut Statistik erkranken in Deutschland jährlich 4440 Frauen neu an einem Zervixkarzinom [19], die Zahl der Todesfälle wird vom Statistischen Bundesamt für 2004 mit 1660 Fällen angegeben.

In der Altersgruppe der 20- bis 25-jährigen Frauen ist in Abhängigkeit von der sexuellen Aktivität die Häufigkeit der HPV-Infektion mit ca. 50\% anzunehmen.

Daraus wird ersichtlich, warum das Zervixkarzinom bei 15- bis 44-jährigen Frauen in der Krankheitshäufigkeit an dritter Stelle der Karzinome steht [19].

\section{Etwa $30 \%$ der HPV-abhängigen Karzinome in Europa finden sich bei Männern [20].}

Es besteht eine lebenslange Exposition für HP-Viren sowohl für die Gruppe der High-Risk-Typen als auch für die Low-Risk-Typen 6 und 11, die Condylomata acuminata verursachen [21].

\section{Impfstoffe}

Zur primären Prävention einer HPV-Infektion stehen in Deutschland derzeit noch 3 Impfstoffe zur Verfügung, ein bivalenter, ein tetravalenter und seit 2016 ein nonavalenter Impfstoff, der den tetravalenten Impfstoff ablösen wird. Der bivalente Impfstoff enthält die Antigentypen HPV 16 und 18, der tetravalente zusätzlich HPV 6 und 11 und der nonavalente Impfstoff enthält die Typen 6, 11, 16, 18, 31, 33, 45, 52 und 58.

Alle 3 HPV-Impfstoffe sind nichtinfektiöse, sog. virusähnliche Partikel-Impfstoffe (VLP). Sie dürfen ab einem Alter von 9 Jahren angewendet werden.

Der tetravalente sowie der nonavalente HPV-Impfstoff sind für den Einsatz bei weiblichen und männlichen Personen zugelassen, der bivalente HPV-Impfstoff nur 
für den Einsatz bei Mädchen und Frauen. Es handelt sich um präventive und keine therapeutischen Impfstoffe, d. h. bestehende Infektionen oder Erkrankungen werden durch sie nach heutiger Kenntnis nicht beeinflusst.

\section{Impfempfehlung}

Das von der STIKO angegebene Impfziel besteht in der Reduktion der Krankheitslast durch das Zervixkarzinom. Die Impfung ist eine Standardimpfung für Mädchen im Alter von 9-14 Jahren und soll spätestens bis zum 18. Lebensjahr erfolgt sein.

Für Jungen ist nach Meinung aller Experten eine ebensolche Impfempfehlung der STIKO überfällig, bislang ist die HPV-Impfung in Deutschland aber nur von der Sächsischen Impfkommission (SIKO) öffentlich empfohlen.

Ausdrücklich empfiehlt die STIKO, die HPV-Impfung auf Basis der Impfstoffzulassungen in ärztlicher Verantwortung auch über die Standardempfehlungen hinaus anzuwenden.

Altersmäßig ist die Impfung nicht nach oben begrenzt und sowohl der tetravalente als auch der nonavalente Impfstoff sind für Jungen und Männer zugelassen. Einige Krankenkassen übernehmen die Kosten für Frauen bis zum Alter von 26 Jahren oder als Rezidivprophylaxe nach operativer Therapie einer zervikalen KarzinomVorstufe.

\section{Grundimmunisierung}

Bereits mit bi- oder tetravalentem Impfstoff begonnene Impfserien sollen solange mit demselben Impfstoff fortgesetzt und beendet werden, wie er verfügbar ist [22].

Neue Impfserien mit dem nonavalenten Impfstoff werden im Alter von 9-14 Jahren im 2-Dosen-Schema, ab dem Alter von 15 Jahren im 3-Dosen-Schema durchgeführt. Maßgeblich für das jeweilige Schema ist das Alter bei der ersten Impfdosis (Tab. 13).

\section{Schutzdauer}

Die Langzeitüberwachung zeigt bisher über 10 Jahre keine Abnahme der Schutzwirkung. Experten gehen deshalb von einer noch viel längeren Schutzdauer aus.

\section{Auffrischimpfungen}

Bislang keine erforderlich. Sobald bei den nach Impfung kontinuierlich überwachten Frauen Signale von Impfdurchbrüchen erkennbar würden, müsste über eine Auffrischimpfung entschieden werden.

\section{Iipp für die Praxis}

Da zu Beginn der HPV-Impfungen durch Medienberichte und auch Einlassungen von Wissenschaftlern eine Verunsicherung der Öffentlichkeit, aber auch von Ärzten stattfand, ist jetzt eine umfassende Aufklärung aller infrage kommenden Patienten wichtig. Nach mehr als 260 Millionen angewandten HPV-Impfdosen bestehen weder bez. der Sicherheit noch der Wirksamkeit und Verträglichkeit Zweifel. Alle Mädchen im Alter von 9-17 Jahren sollten zulasten der Krankenkassen geimpft, allen Jungen im gleichen Alter sollte die Impfung als individuelle Gesundheitsleistung empfohlen werden.

\section{Meningokokken C}

\section{Epidemiologie}

Seit 2006 gibt es eine STIKO-Empfehlung zur Impfung aller Kinder und Jugendlichen bis zum 18. Lebensjahr gegen Meningokokken C. In Deutschland erkranken heute trotz der Impfung noch immer um die 300 Menschen jährlich, etwa 70\% durch den Serotyp B und 30\% durch den Serotyp C. Etwa 10\% der Erkrankten sterben.

$40 \%$ aller Meningokokken-Erkrankten sind Kinder unter 4 Jahren.

\section{Impfstoffe}

Drei Meningokokken-C-Konjugatimpfstoffe sind verfügbar.

Obwohl seit einigen Jahren ein Impfstoff gegen Meningokokken B zugelassen ist, wurde bislang von der STIKO hierfür noch keine Empfehlung zur Standardimpfung ausgesprochen. Die SIKO hingegen hat die Impfung bereits öffentlich für Kinder empfohlen.

Konjugierte Kombinationsimpfstoffe gegen Meningokokken A, C, W135 und Y sind speziellen Indikationen vorbehalten (Tab. 14). 
Tabelle 13

Impfschemata der 3 verfügbaren HPV-Impfstoffe.

\begin{tabular}{|c|c|c|}
\hline Impfstoff & Termin & Zeitschema \\
\hline Cervarix $^{\circledR}$ & 1. Impftermin & heute \\
\hline \multicolumn{3}{|l|}{ 9-14 Jahre } \\
\hline \multirow[t]{2}{*}{ ab 15 Jahre } & 2. Impftermin & 1 (2) Monate nach 1. Impfung \\
\hline & 3. Impftermin & $6(3-10)$ Monate nach 2. Impfung \\
\hline Gardasil $^{\circledR}$ & 1. Impftermin & heute \\
\hline 2-Dosen-Schema $(0 / 6)$ & 2. Impftermin & 6 Monate nach 1. Impfung \\
\hline \multicolumn{3}{|l|}{ 9-13 Jahre } \\
\hline 3-Dosen-Schema $(0 / 2 / 6)$ & 1. Impftermin & heute \\
\hline \multirow[t]{2}{*}{ ab 14 Jahre } & 2. Impftermin & 2 (1) Monate nach 1. Impfung \\
\hline & 3. Impftermin & $6(3-10)$ Monate nach 2. Impfung \\
\hline Gardasil $^{\circledR} 9$ & 1. Impftermin & heute \\
\hline 2-Dosen-Schema $(0 / 5-13)$ & 2. Impftermin & 5-13 Monate nach 1. Impfung \\
\hline \multicolumn{3}{|l|}{ 9-14 Jahre } \\
\hline 3-Dosen-Schema $(0 / 2 / 6)$ & 1. Impftermin & heute \\
\hline
\end{tabular}

\section{Tabelle 14}

Indikationen für eine Meningokokkenimpfung gemäß STIKO.

\begin{tabular}{|c|c|c|}
\hline Art & Kategorie & Personengruppen \\
\hline $\begin{array}{l}\text { Standardimpfung } \\
\text { gegen Meningokokken C }\end{array}$ & $\mathrm{S}$ & $\begin{array}{l}\text { - einmalige Impfung aller Kinder im 2. Lebensjahr } \\
\text { - Nachholen der Impfung bis zum 18. Geburtstag }\end{array}$ \\
\hline \multirow[t]{3}{*}{$\begin{array}{l}\text { Indikationsimpfung gegen } \\
\text { Meningokokken A,C,W135,Y }\end{array}$} & 1 & $\begin{array}{l}\text { 1. Gesundheitlich gefährdete Personen mit angeborener oder erworbener Immundefizienz bzw. -suppression mit } \\
\text { T- und/oder B-zellularer Restfunktion, insbesondere Komplement-/Properdindefekte, Hypogammaglobulinämie; } \\
\text { Asplenie }\end{array}$ \\
\hline & B & 2. gefährdetes Laborpersonal \\
\hline & $\mathrm{R}$ & $\begin{array}{l}\text { - Reisende in Länder mit epidemischem/hyperendemischem Vorkommen, besonders bei engem Kontakt zur } \\
\text { einheimischen Bevölkerung (z. B. Entwicklungshelfer, Katastrophenhelfer, medizinisches Personal, bei Langzeit- } \\
\text { aufenthalt); dies gilt auch für Aufenthalte in Regionen mit Krankheitsausbrüchen und Impfempfehlung für die } \\
\text { einheimische Bevölkerung (WHO- und Länderhinweise beachten). } \\
\text { - vor Pilgerreise nach Mekka (Hadsch) } \\
\text { - Schüler/Studenten vor Langzeitaufenthalten in Ländern mit empfohlener allgemeiner Impfung für Jugendliche } \\
\text { oder selektiver Impfung für Schüler/Studenten }\end{array}$ \\
\hline $\begin{array}{l}\text { Indikationsimpfung gegen } \\
\text { Meningokokken B }\end{array}$ & I/B & $\begin{array}{l}\text { Obige Gruppen } 1 \text { und } 2 \text { sollten zusätzlich zur tetravalenten Impfung eine Impfung gegen Meningokokken B } \\
\text { erhalten. } \\
\text { - Darüber hinaus legt es die STIKO in die Entscheidung und Verantwortung des Arztes, wer gegen Meningo- } \\
\text { kokken B geimpft werden soll. }\end{array}$ \\
\hline
\end{tabular}


Impfempfehlung

Die STIKO empfiehlt die einmalige Impfung gegen Meningokokken der Serogruppe $C$ mit einem konjugierten Meningokokken-C-Impfstoff für alle Kinder im 2. Lebensjahr.

Eine fehlende Impfung sollte bei allen Jugendlichen bis zum 18. Geburtstag nachgeholt werden.

Über diese Standardimpfung hinaus empfiehlt die STIKO die einmalige Indikationsimpfung mit tetravalentem Impfstoff für Personen mit bestimmten Vorbedingungen (s. Tab. 14).

Ob zusätzlich zur Impfung mit tetravalentem Impfstoff auch eine Impfung gegen Meningokokken B erfolgen soll, überlässt die STIKO der verantwortlichen Entscheidung des Arztes.

Dosierung und Notwendigkeit einer Auffrischimpfung sind den jeweiligen Fachinformationen der Impfstoffe zu entnehmen.

\section{Tipp für die Praxis}

Da viele der Jugendlichen, die heute die Frauenarztpraxis zur HPV-Impfung oder anderen Anlässen aufsuchen, die Meningokokken-C-Impfung noch nicht in der Kindheit erhalten haben, besteht hier ein hoher Bedarf an Nachholimpfungen. Die MeningokokkenB-Impfung wird bereits von der SIKO seit 2014 allen Kindern und Jugendlichen bis 17 Jahre empfohlen. Auch ohne STIKO-Empfehlung ist es sinnvoll, die Impfung als IGe-Leistung in dieser Altersgruppe anzubieten.

\section{Grundimmunisierung}

Bei der Meningokokken-C-Impfung wird einmalig eine Einzeldosis verabreicht. Bei der tetravalenten Impfung gegen Meningokokken A, C, W, Y wird ebenfalls eine Impfdosis erforderlich.

Die Meningokokken-B-Impfung wird mit 2 Einzeldosen im Abstand von 1-2 Monaten grundimmunisiert. Säuglinge von 2-5 Monaten sollen eine 3. Impfdosis erhalten.

\section{Schutzdauer}

Nach erfolgter Grundimmunisierung bleibt ein jahrelanger Schutz erhalten.

\section{Auffrischung}

Für die Meningokokken-C-Impfung ist keine Auffrischung vorgesehen. Zu Wiederholungsimpfungen bei anderen Meningokokkenimpfungen ist die Fachinformation des jeweiligen Impfstoffs zu beachten.

\section{Postexpositionsprophylaxe}

Personen mit engem Kontakt zu einem Erkrankten sollten neben der empfohlenen Chemoprophylaxe eine Impfung mit einem entsprechenden MeningokokkenImpfstoff erhalten.

\section{Pneumokokken}

\section{Epidemiologie}

Pneumokokken sind bekapselte Bakterien, von denen es mehr als 90 Antigentypen gibt. Sie sind oft Bestandteil der residenten Flora des Oropharynx und nicht obligat pathogen. Erst eine Schwächung der lokalen oder systemischen Immunabwehr begünstigt die Ausbreitung der Infektion. Besonders gefährdet sind: Neugeborene und Personen ab dem 60. Lebensjahr, nach vorausgegangenen Infektionen (z. B. Influenza), bei Grunderkrankungen (wie z.B. Asthma, COPD oder Diabetes mellitus), nach Operationen sowie bei Immundefizienz oder medikamentöser Immunsuppression.

Die Infektionen verlaufen meist asymptomatisch. Pneumokokken sind Ursachen von nichtinvasiven (Otitis media, Sinusitis) oder invasiven Erkrankungen (Pneumonie mit Bakteriämie, Meningitis).

Die jährliche Inzidenz invasiver Pneumokokkenerkrankungen steigt mit dem Alter.

Die WHO meldet weltweit bis zu 1,6 Millionen Todesfälle durch invasive Pneumokokkenerkrankungen. Nach Schätzungen des Robert Koch-Institutes versterben in Deutschland jährlich 12000 Menschen an Pneumokokken-Pneumonien [23]. 
Eine Zunahme der Antibiotikaresistenz (Penicillin) bei Pneumokokken wird beschrieben.

\section{Impfstoffe}

Für die Impfung stehen 2 Impfstoffe zur Verfügung: Ein 23-valenter Polysaccharid-Impfstoff (PPSV23) für Erwachsene, Jugendliche und Kinder ab dem 2. Lebensjahr und ein 13-valenter Konjugat-Impfstoff (PCV13) für Erwachsene, Jugendliche und Kinder ab der 6. Lebenswoche.

\section{Impfempfehlung}

Die Pneumokokkenimpfung ist Standardimpfung in den ersten 2 Lebensjahren und für Personen ab 60 Jahre. Die Indikationen außerhalb dieses Altersbereiches sind in Tabelle 15 zusammengefasst. Die STIKO hält derzeit in ihren Empfehlungen für Erwachsene am 23-valenten Impfstoff fest, während die Schutzimpfungsrichtlinie keine Festlegung auf einen bestimmten Impfstoff vornimmt. Die Sächsische Impfkommission rät bereits bei der einmaligen Standardimpfung über 60-Jähriger generell zum 13-valenten Konjugatimpfstoff.

Im gynäkologischen Fachgebiet sind für die Pneumokokkenimpfung vorzusehen:

- Frauen vor immunsuppressiver Behandlung (z.B. Chemotherapie wegen Mammakarzinom)

- Frauen mit chronischen Herz- und Kreislauf-Erkrankungen oder Erkrankungen der Atmungsorgane

- Diabetikerinnen

\section{Grundimmunisierung}

Als Standardimpfung ab 60 Jahre gemäß STIKO eine Einzeldosis Polysaccharidimpfstoff PPSV23. Neugeborene siehe Tab. 15. Für Indikationsimpfungen empfiehlt die STIKO folgendes Vorgehen:

- Gruppe 1 und 3: Sequenzielle Impfung mit 1 Dosis PCV13 gefolgt von 1 Dosis PPSV23 nach 6-12 Monaten, wobei PPSV23 erst ab einem Alter von 2 Jahren gegeben werden soll.

- Gruppe 2: Bei Personen ab 16 Jahren eine Einzeldosis PPSV23, von 2-15 Jahren sequenzielle Impfung mit 1 Dosis PCV13 gefolgt von 1 Dosis PPSV23 nach 6-12 Monaten.

- Berufliche Indikation: 1 Dosis PPSV23.

\section{Tabelle 15}

\section{Indikationen zur Pneumokokkenimpfung gemäß STIKO.}

\begin{tabular}{|c|c|}
\hline Art & Kategorie \\
\hline $\begin{array}{l}\text { Standard- } \\
\text { impfung }\end{array}$ & $S$ \\
\hline $\begin{array}{l}\text { Indikations- } \\
\text { impfung }\end{array}$ & I \\
\hline
\end{tabular}

- für alle Neugeborenen (3-malige Impfung mit Konjugatimpfstoff bis 14. Lebensmonat, Frühgeborene 4 Impfdosen)

- für alle Personen ab 60 Jahre (einmalige Impfung mit 23-valentem Polysaccharid-Impfstoff)

impfung

- Kinder ab 2 Jahren, Jugendliche und Erwachsene mit erhöhter gesundheitlicher Gefährdung infolge einer Grundkrankheit:

1. Angeborene oder erworbene Immundefekte bzw. Immunsuppression, wie z. B.:

- T- und B-Zell-Defizienz

- Hypogammaglobulinämie, Komplement- und Properdindefekte

- bei funktioneller oder anatomischer Asplenie

- bei Sichelzellenanämie

- bei Krankheiten der blutbildenden Organe

- bei neoplastischen Krankheiten

- bei HIV-Infektion

- nach Knochenmarktransplantation

- vor Organtransplantation und vor Beginn einer immunsuppressiven Therapie

2. Sonstige chronische Krankheiten, wie z. B.:

- Herz-Kreislauf-Krankheiten oder Krankheiten der Atmungsorgane (inkl. Asthma und COPD)

- Diabetes mellitus oder andere Stoffwechselkrankheiten

- chronische Nierenkrankheiten/nephrotisches Syndrom

- neurologische Krankheiten, z. B. Zerebralparesen oder Anfallsleiden

3. Anatomische und Fremdkörper-assoziierte Risiken für Pneumokokken-Meningitis, wie z. B.

- Liquorfistel

- Cochlea-Implantat

B

berufliche Tätigkeiten wie Schweißen und Trennen von Metallen, die zu einer Exposition gegenüber Metallrauchen einschließlich metalloxidischen Schweißrauchen führen

Wiederholungs- A impfung

Aufgrund der begrenzten Dauer des Impfschutzes soll die Impfung mit PPSV23 in allen 3 Risikogruppen mit einem Mindestabstand von 6 Jahren wiederholt werden.

\section{Schutzdauer}

Der Impfschutz besteht für die Dauer von bis zu 6 Jahren. 


\section{Auffrischung}

Alle Personen, die zu Risikogruppen in Tabelle 15 gehören, sollen alle 6 Jahre eine Impfung mit PPSV23 erhalten. Dies gilt auch für die berufliche Indikation, solange die Exposition andauert.

\section{Influenza}

\section{Epidemiologie}

In Deutschland zählt der Tod durch Influenza bzw. bakteriellen Superinfektionen der Atemwege zu den häufigsten durch Impfung vermeidbaren Todesfällen. Jährlich führt die Influenzawelle weltweit zu mehr als 1-2 Millionen Todesfällen. In unregelmäßigen Abständen kommt es immer wieder zu schweren Grippeepidemien, die eine Übersterblichkeit von über 30000 Personen zur Folge haben.

Die WHO beobachtet das Auftreten von Grippeausbrüchen weltweit und gibt jedes Jahr eine Empfehlung zur Zusammensetzung des neuen Grippeimpfstoffs heraus. Aber innerhalb der Grippesaison kommt es bereits zu Veränderungen des Subtyps (Antigendrift) einer der Gründe, weshalb immer Fälle von Grippe auch bei aktuell Geimpften auftreten können.

\section{Jährliche Teilnahme an der Grippeimpfung sichert den Geimpften ein immer größer werdendes Abwehrspektrum.}

Gefürchtet wird das Auftreten neuer Subtypen (Antigenshift) durch Vermischung von Menschen-, Vogelund Schweineviren in einem befallenen Organismus mit nachfolgender Möglichkeit einer Übertragung von Mensch zu Mensch. Erinnert sei dabei an das Auftreten eines neuen A-H1N1-Virus 2009, das einen weltweiten Kampf zur Eindämmung einer Pandemie zur Folge hatte. In der Nachschau hat die sog. „Schweinegrippe“ weltweit mehr als 200000 Todesopfer gefordert [24].

\section{Impfstoffe}

Jährlich wird wegen der Virusvariabilität ein neuer tri- oder tetravalenter Totimpfstoff von verschiedenen Herstellern für die jeweilige Saison angeboten.
Ein nasaler Lebendimpfstoff für Kinder sollte nach STIKO im Alter von 2-6 Jahren bevorzugt zum Einsatz gelangen [3]. Er ist bis zum Alter von 17 Jahren zugelassen.

Für Personen ab 65 Jahre steht ein adjuvantierter Grippeimpfstoff zur Verfügung. Eine entsprechende Empfehlung im aktuellen Impfkalender der STIKO zur Anwendung adjuvantierter Grippeimpfstoffe findet sich nicht. Allerdings hatte bereits 2003 die STIKO geraten, dass an Personen über 65 Jahre wegen der „möglicherweise eingeschränkten Immunantwort adjuvantierte Influenza-Impfstoffe zu verabreichen sind ..." [25].

Seit der Saison 2013/2014 steht ein tetravalenter Grippeimpfstoff zur Verfügung. Dieser Impfstoff enthält neben den 2 A-Antigenen und dem einen B-Antigen in den üblichen Grippeimpfstoffen einen weiteren B-Antigen-Typ, da in den letzten Jahren häufig mehrere B-Virus-Typen im Umlauf waren. So enthielt in der Saison 2015/2016 der trivalente Impfstoff nicht den Influenzavirustyp, der Hauptverursacher von Influenza war. Im tetravalenten Impfstoff hingegen war er enthalten. Die abgeschlossenen Rabattverträge mit den Krankenkassen ließen aber ein Umsteigen auf den wirksamen Impfstoff und eine differenzierte Anwendung der Influenza-Impfstoffe nicht zu.

Die Zusammensetzung des Influenza-Impfstoffs variiert von Jahr zu Jahr.

\section{Impfempfehlung}

Die Influenzaimpfung ist Standardimpfung für alle Personen ab dem Alter von 60 Jahren, darüber hinaus Indikationsimpfung für alle Schwangeren und chronisch Kranken sowie für Bewohner von Alters- und Pflegeheimen und Personen mit häufigem Publikumsverkehr in beruflicher Situation (STIKO-Kategorie B). Medizinisches Personal und Personen mit Kontakt zu Risikopersonen und Möglichkeit zur Übertragung der Influenza auf diese Personengruppe sollten nach Empfehlung der STIKO ebenfalls über einen Impfschutz verfügen (Tab. 16). Da die Influenza die häufigste reiseassoziierte Erkrankung darstellt, sollte die Impfung allen Reisenden angeboten werden. 


\section{Tipp fur die Praxis}

Die besten Monate für die Influenzaimpfung sind September und Oktober. Nicht erst warten bis die Grippesaison beginnt.

Wird nach einer bereits erfolgten Infektion geimpft, kann der Verlauf deutlich schwerer, jedoch nie wegen der Impfung bedrohlich ausfallen. Vorurteile gegen die Grippeimpfung sind u. a. auch hierauf zurückzuführen.

\section{Grundimmunisierung}

Jährlich eine Impfdosis mit aktueller, von der WHO empfohlener Antigenkombination.

\section{Schutzdauer}

Die Impfung erzeugt nur gegen diejenigen Virustypen eine Schutzwirkung, die als Antigen im saisonalen Impfstoff sind. Aber auch andere Virustypen können im Umlauf sein. Allerdings soll sich auch durch Influenzaimpfungen ein gewisses immunologisches Gedächtnis ausbilden, sodass die Infektionsabwehr durch jährliche Impfungen immer breiter wird.

Auch Geimpfte können wegen der hohen Variabilität der Viren dennoch an Grippe erkranken.

Von der A-H1N1-Pandemie 2009 waren in der Mehrzahl jüngere - also selten oder nicht vorgeimpfte Personen betroffen; 85\% der Todesopfer waren unter 65 Jahre alt.

\section{Auffrischung}

Jährliche Wiederimpfung erforderlich.

\section{Impfungen bei besonderen Vorbedingungen}

\section{Vor, während und nach einer Schwangerschaft}

Aus ethischen und rechtlichen Gründen werden Schwangere und möglicherweise schwangere Frauen von der Teilnahme an Zulassungsstudien eines Impfstoffs grundsätzlich ausgeschlossen.

\section{Tabelle 16}

Indikationen für die jährliche Influenzaimpfung gemäß STIKO.

\section{Kategorie}

Standard- S für alle Personen ab 60 Jahre

impfung

Indikations- ।

impfung

I

a alle Schwangeren ab 2. Trimenon, bei erhöhter gesundheitlicher Gefährdung infolge eines Grundleidens schon im 1. Trimenon

- Kinder, Jugendliche und Erwachsene mit erhöhter gesundheitlicher Gefährdung infolge eines Grundleidens, wie $\mathrm{z}$. B.

- chronische Krankheiten der Atmungsorgane (inklusive Asthma und COPD)

- chronische Herz-Kreislauf-, Leber- und Nierenkrankheiten

- Diabetes und andere Stoffwechselkrankheiten

- chronische neurologische Krankheiten, z. B. Multiple Sklerose mit durch Infektionen getriggerten Schüben

- Personen mit angeborenen oder erworbenen Immundefekten mit T- und/oder B-zellularer Restfunktion

- HIV-Infektion

- Bewohner von Alters- oder Pflegeheimen

- Kontaktpersonen von Risikopersonen

Berufliche Indikation: Personen mit erhöhter Gefährdung, z. B. medizinisches Personal, Personen in Einrichtungen mit umfangreichem Publikumsverkehr sowie Personen, die als mögliche Infektionsquelle für von ihnen betreute Risikopersonen fungieren können

Die Erfahrung hat gezeigt, dass Totimpfstoffe bei Notwendigkeit auch in der Schwangerschaft erlaubt, dagegen Lebendimpfstoffe wegen eines theoretischen Risikos durch abgeschwächte Viren in der Schwangerschaft verboten sind. Allerdings hat es auch hier weltweit keine Schadensmeldungen gegeben, weshalb eine versehentliche Lebendimpfung kurz vor oder während der Schwangerschaft kein Grund für Besorgnis oder gar einen Schwangerschaftsabbruch ist [26]. In den Fachinformationen der Lebendimpfstoffe ist inzwischen deshalb nur noch ein Monat Abstand zu einem Schwangerschaftseintritt angeraten.

Nach Ansicht des Paul-Ehrlich-Institutes (PEI) ist der Einsatz ausgewählter Impfstoffe bei Frauen mit Kinderwunsch und bei Schwangeren ein wichtiger Bestandteil der medizinischen Betreuung für Mutter, Ungeborenes und Neugeborenes. 


\section{Tabelle 17}

Impfungen vor und in einer Schwangerschaft sowie in der Stillzeit.

\begin{tabular}{|c|c|c|c|}
\hline & vor der Schwangerschaft & in der Schwangerschaft & nach der Schwangerschaft \\
\hline Impfziel & alle Impflücken schließen & $\begin{array}{l}\text { notwendige Impfungen } \\
\text { durchführen }\end{array}$ & $\begin{array}{l}\text { fehlende Impfungen } \\
\text { vervollständigen }\end{array}$ \\
\hline \multicolumn{4}{|l|}{ Impfung gegen } \\
\hline Tdap, Tdap-IPV & $\begin{array}{l}\text { nach Lebendimpfstoff } \\
4 \text { Wochen Abstand }\end{array}$ & Tetanus (Tdap/IPV) & \multirow{7}{*}{$\begin{array}{l}\text { In Wochenbett und Stillzeit } \\
\text { sind alle Impfungen erlaubt, } \\
\text { auch Lebendimpfstoffe } \\
\text { neben Totimpfstoffen ... } \\
\text { außer Gelbfieberimpfung, } \\
\text { wenn gestillt wird. }\end{array}$} \\
\hline Influenza & zur Schwangerschaft; & Influenza & \\
\hline Hepatitis B & $\begin{array}{l}\text { Eintritt einer } \\
\text { Schwangerschaft }\end{array}$ & Hepatitis B & \\
\hline MMR & $\begin{array}{l}\text { in dieser Zeit oder } \\
\text { versehentliche Impfung } \\
\text { in der Schwangerschaft }\end{array}$ & nicht erlaubt & \\
\hline Varizellen & ohne Konsequenz & nicht erlaubt & \\
\hline FSME & & falls erforderlich & \\
\hline Reiseimpfungen & & falls erforderlich & \\
\hline
\end{tabular}

Kontaktpersonen impfen

\section{Tipp für die Praxis}

Wenn Impfungen in der Schwangerschaft erforderlich sind, sollten zur Vermeidung unnötiger Diskussionen aufschiebbare Impfungen möglichst erst nach der 12. SSW geimpft werden.

Falls im 1. Trimenon geimpft werden muss, sollte die Schwangere darauf hingewiesen werden, dass in den ersten Monaten noch ein erhöhtes Risiko für natürliche Aborte besteht, eine Impfung aber einen solchen keinesfalls verursachen kann.

Schwangeren sollte grundsätzlich von Reisen in Gefährdungsgebiete abgeraten werden. Ist eine solche Reise aber nicht verschiebbar, können auch notwendige Reiseimpfungen während der Schwangerschaft erfolgen.

Grundsätzlich sollte in der Schwangerschaft so viel wie nötig und so wenig wie möglich geimpft werden. Jede notwendige Impfung zum Schutz der Schwangeren aber muss und darf verabreicht werden, außer Lebendimpfungen gegen MMR und Varizellen (s. Tab. 17).

Auch für Schwangere gilt: Nichtimpfen ist gefährlicher als Impfen!

\section{Tipp für die Praxis}

Kontaktpersonen von Schwangeren und Neugeborenen sollten über einen vollständigen Impfschutz verfügen, der besonders auch Schutz vor Masern, Varizellen, Pertussis und Influenza einschließt. Eine Schwangerschaft in der Umgebung einer zu impfenden Person ist keine Kontraindikation für eine Impfung.

\section{Chronisch Kranke}

Je älter und kranker ein Mensch ist, desto mehr Schutz vor Infektionen benötigt er. Chronisch Kranke haben bei Infektionen ein erhöhtes Risiko für Komplikationen. Ihr Immunsystem ist zudem geschwächt und die Immunantwort auf Impfungen ist reduziert.

Alle Impfungen sind erlaubt, die Impfantwort kann ggf. durch adjuvantierte Impfstoffe verbessert werden.

Neben allen Standardimpfungen sind besonders Impfungen gegen Pertussis, Influenza und Pneumokokken wichtig. Nicht vergessen werden sollte die Impfung von Kontaktpersonen. 


\section{Multiple Sklerose}

Alle Standard- und Indikationsimpfungen mit Totimpfstoffen dürfen nach Empfehlungen der National MS-Society der USA und der Deutschen Multiple Sklerose Gesellschaft verabreicht werden [27].

Die Impfungen sollten möglichst im schubfreien Intervall außerhalb einer Kortikoidtherapie erfolgen.

Unverzichtbar ist die Influenzaimpfung, da die echte Grippe häufig einen Schub auslöst.

Bei fehlendem Schutz vor Masern, Mumps, Röteln oder Varizellen sollte vor der Risiko-Nutzen-Abwägung einer Lebendimpfung zunächst die Antikörperkontrolle erfolgen. Bei Seropositivität kann dann auf eine Impfung verzichtet werden. Eine Überprüfung aller beim PEI von 2001-2009 eingegangenen Meldungen zu MMR-Impfungen erbrachte keine schweren Nebenwirkungen und keine MS-Auslösung durch Impfung [28].

\section{Immundefiziente}

Zunehmend kommen Therapeutika zum Einsatz (Rheuma, Psoriasis, Multiple Sklerose, Karzinome u. a. m.), die zu einer Immunsuppression führen. Bei Immundefizienz ist die Anwendung von Lebendimpfstoffen wegen der Gefahr einer schweren vakzineassoziierten Erkrankung bis hin zur tödlichen Komplikation kontraindiziert und die Immunogenität von Totimpfstoffen oftmals stark herabgesetzt. Deshalb sollten alle erforderlichen Impfungen möglichst 2-4 Wochen vor Beginn einer solchen Therapie erfolgt sein. Auch hier wäre ein lebenslang begleitender kompletter Impfschutz die beste Schutzvoraussetzung für eine im späteren Alter eventuell notwendig werdende immunsupprimierende Behandlung.

Wie bei den oben erwähnten Personen mit chronischen Erkrankungen sind neben allen Standardimpfungen besonders Impfungen gegen Pertussis, Influenza und Pneumokokken wichtig sowie ein sicherer Impfschutz bei allen Kontaktpersonen.

\section{Antikoagulanzientherapie}

Intramuskuläre Injektionen sind gemäß Fachinformation von Antikoagulanzien mit dem Wirkstoff Phenprocoumon oder Warfarin verboten. Neue Antikoagulanzien mit dem Wirkstoff Apixaban, Dabigatran oder Rivaroxaban enthalten diesen Hinweis nicht. Bislang wurden die Impfungen subkutan verabreicht, was oft schmerzhaft war und bei Adsorbatimpfstoffen stärkere Lokalreaktionen hervorrief. Intramuskuläre Impfungen können aber nach Aufklärung des Patienten über eventuelle Blutungen und Hämatome durchgeführt werden (PEI 2009, Arzneitelegramm 2013, DGK 2013, KVBW 2014) [29]. Um die Zahl der Injektionen zu vermindern, sollten bevorzugt Kombinationsimpfstoffe zum Einsatz gelangen.

\section{Tipp für die Praxis}

Patienten unter Antikoagulanzientherapie sollten zur Vermeidung mehrfacher Injektionen möglichst Kombinationsimpfstoffe erhalten. Nach entsprechender Aufklärung über Blutungen und Hämatome darf eine intramuskuläre, langsame Applikation des Impfstoffs mit dünner 23er-Nadel erfolgen. Nachfolgend sollte eine Kompression der Injektionsstelle über mindestens 10 Minuten durchgeführt werden.

\section{Kernaussagen}

Impfungen sind die wichtigste Präventionsmaßnahme. Deshalb gehört das Angebot von Schutzimpfungen in jede verantwortungsvolle Frauenarztpraxis. Keine von uns betreute Frau sollte mehr wegen einer unterlassenen Impfung erkranken.

Wenn wir uns bemühen, allen Jugendlichen bis zum 18. Geburtstag die nach STIKO-Impfplan erforderlichen Impfungen zu ermöglichen und weiterhin für regelmäßige Auffrischungen des Impfschutzes zu sorgen, brauchen wir nicht mehr über fehlenden Impfschutz bei den ersten Sexualkontakten, bei Schwangeren, Immundefizienten, chronisch Kranken und Karzinompatienten nachzudenken: Durch unsere Aufmerksamkeit haben wir unseren Patienten einen aktuellen Impfschutz durch alle Lebensphasen garantiert. 


\section{Über den Autor}

\section{Michael Wojcinski}

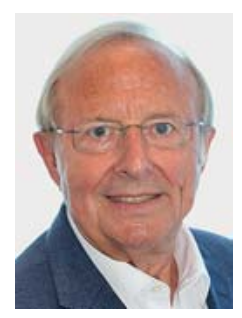

Dr. med. Jahrgang 1944. Medizinstudium in Münster, Staatsexamen und Promotion 1971, Approbation 1972. 1973-1974 Stabsarzt an der Sanitätsakademie der Bundeswehr (München), 1974-1978 Weiterbildung zum Facharzt für Frauenheilkunde und Geburtshilfe (Bielefeld), 1978-2012 Niederlassung in eigener Praxis in Bielefeld. Schwerpunkt: Prävention, Impfmedizin, Reproduktionsmedizin, Ambulante Operationen, Zytologisches Labor. 1986-2010 Stellvertretender Landesvorsitzender von Westfalen-Lippe des Berufsverbandes der Frauenärzte e. V. (BVF). Seit 1998 Sprecher der Arbeitsgemeinschaft „Impfen in der Gynäkologie“ des BVF, seit 2006 Leiter des CMV-Lenkungskreises des BVF, seit 2007 Mitglied der Initiative Connatale Cytomegalie (ICON). 2007 Verleihung des Fortbildungspreises des BVF und 2010 Verleihung der Ehrenmitgliedschaft im BVF.

\section{Interessenkonflikt}

Der Autor gibt an, dass kein Interessenkonflikt besteht.

\section{Korrespondenzadresse}

Dr. med. Michael Wojcinski

Frauenarzt, Sprecher der AG Impfen des Berufsverbandes der Frauenärzte e. V.

Schulstr. 16

82490 Farchant/Kreis Garmisch-Partenkirchen

E-Mail: dr@wojcinski.de

\section{Literatur}

1 Poetthko-Müller C, Schmitz R, Abt. für Epidemiologie und Gesundheitsmonitoring, Robert Koch-Institut, Berlin. Impfstatus von Erwachsenen in Deutschland - Ergebnisse der Studie zur Gesundheit Erwachsener in Deutschland (DEGS1). Bundesgesundheitsblatt 2013; 56: 845-857

2 Forsa-Institut. Einstellungen, Wissen und Verhalten der Allgemeinbevölkerung zu Hygiene und Infektionsschutz, Studie des Forsa-Instituts im Auftrag der BZGA; 2013

3 Robert Koch-Institut. Empfehlungen der Ständigen Impfkommission (STIKO). Epidemiologisches Bulletin 2016; Nr. 34

4 BGH-Urteil VI ZR 48/99 vom 15.02.2000

5 Beytout J, Launay O, Guiso N et al. Safety of TdaP-IPV given 1 month after Td-IPV booster in healthy young adults: a placebocontrolled trial. Human Vaccines 2009; 5: 315-321

6 Hülße C. Krefeld-Rostocker Erwachsenen-Studie zur Hustengenese. Rostock; 2007
7 http://www.cdc.gov/media/releases/2012/a1024_TdaP_immunization.html; Stand: 30.08.2016

8 Billingsley M. Pregnant women in UK are offered whooping cough vaccine to protect newborns. Br Med J 2012; 345: e6594

9 Robert Koch-Institut. Epidemiologisches Bulletin 2016; Nr. 4

10 Poethko-Müller C, Zimmermann R, Hamouda O et al. Die Seroepidemiologie der Hepatitis A, B und C in Deutschland. Ergebnisse der Studie zur Gesundheit Erwachsener in Deutschland (DEGS1). Bundesgesundheitsblatt Gesundheitsforschung Gesundheitsschutz 2013; 56: 707-715

11 Robert Koch-Institut. Ratgeber für Ärzte Hepatitis B; Stand 12.01.2012

12 Bortolotti F, Guido M, Bartolacci S et al. Chronic hepatitis B in children after e antigen seroclearance: final report of a 29-year longitudinal study. Hepatology 2006; 43: 556-562

13 Schönberger K, Ludwig MS, Wildner M et al. Epidemiology of subacute sclerosing panencephalitis (SSPE) in Germany from 2003 to 2009: a risk estimation. PLoS One 2013; 8: e68909

14 Hviid A, Rubin S, Mühlemann K. Mumps. Lancet 2008; 371 : 932-944

15 AWMF-Leitlinie „Labordiagnostik schwangerschaftsrelevanter Virusinfektionen“. AWMF-Verzeichnis 2014; Nr. 093/001

16 Richtlinie des Gemeinsamen Bundesausschusses zur Empfängnisregelung und zum Schwangerschaftsabbruch (vormals: Sonstige Hilfen-Richtlinien); zuletzt geändert am 21.07.2011; in Kraft getreten am 15.09.2011, veröffentlicht im Bundesanzeiger 2011; Nr. 139: 3251

17 Hampl M. Gebärmutterhalskrebs und Genitalwarzen. Stuttgart: Thieme; 2009: 3, 8

18 Serrano B, Alemany L, Tous S et al. Potential impact of a nine valent vaccine in human papillomavirus related cervical disease. Infect Agent Cancer 2012; 7: 38

19 Castellsagué $X$ et al. ICO Information Centre on HPV and Cervical Cancer. Human Papillomavirus and Related Diseases in Germany. Premdict edition, Summary Report 2013: 6

20 Hartwig S, Syrjänen S, Dominiak-Felden G et al. Estimation of the epidemiological burden of human papillomavirus-related cancers and non-malignant diseases in men in Europe: a review. BMC Cancer 2012; 12: 30

21 Kjaer SK, Breugelmans G, Munk C et al. The burden of genital warts: a study of nearly 70000 woman from the general female population in the 4 Nordic countries. J Infect Dis 2007; 196: $1447-1454$

22 Robert-Koch-Institut. Epidemiologisches Bulletin 2016; Nr. 16 23 Hülße C, Heiniger U, Hrsg. Ärztemerkblatt Pneumokokken. 4. Ausgabe. Marburg: Deutsches Grünes Kreuz; 2011

24 Simonsen L, Spreeuwenberg P et al. Global mortality estimates for the 2009 influenza pandemic from the GLaMOR Project: a modeling study. PLOS Med 2013; 10: e1001558

25 Robert Koch-Institut. Epidemiologisches Bulletin 2003; Nr. 17

26 Keller-Stanislawski B et al. Safety of immunization during pregnancy: A review of the evidence of selected inactivated and live attenuated vaccines. Vaccine 2014; 32: 7057 -7064

27 http://www.dmsg.de; Stand: 30.08.2016

28 Mentzer D, Meyer H, Keller-Stanislawski B. Sicherheit und Verträglichkeit von monovalenten Masern- und kombinierten Masern-, Mumps-, Röteln- und Varizellenimpfstoffen. Bundesgesundheitsbl 2013; 56: 1253-1259

29 Kassenärztliche Vereinigung Baden-Württemberg. Schwierige Impffragen - kompetent beantwortet. Verordnungsforum 30/ 2014 


\section{CME-Fragen}

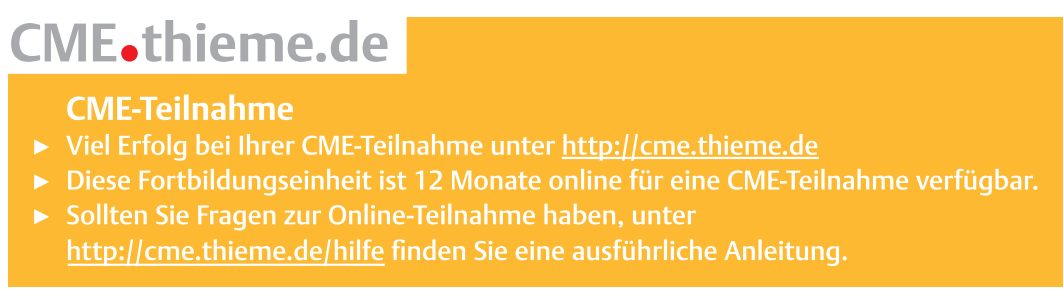

Bei welchen Erwachsenen soll gemäß der STIKO-Empfehlung eine Varizellenimpfung durchgeführt werden?

A bei gebärfähigen Frauen, vor immunsupprimierender Therapie und Patienten mit schwerer Neurodermitis, wenn bei diesen Personen die Seronegativität bestätigt wurde

B bei allen Erwachsenen, die Kontakt zu Neugeborenen haben

C nur bei seronegativen gebärfähigen Frauen

D nur bei Immundefizienten

E gar nicht mehr nach dem 18. Geburtstag

2 Welche Aussage ist bez. einer HPV-Impfung falsch?

A Die Impfung darf als Totimpfstoff auch bei Immundefizienz gegeben werden.

B Sie schützt auch vor Analkarzinomen und deren Vorstufen.

C Die Impfung darf u. a. zeitgleich zur Tetanus, Diphtherie, Pertussis, Polioimpfung gegeben werden.

D Die HPV-Impfung ist nur für weibliche Personen zugelassen.

E Die Impfung ist in der Leistungspflicht der Krankenkassen für weibliche Personen von 9-17 Jahren enthalten.

3 Wann sind Antikörperbestimmungen im Zusammenhang mit einer Impfung sinnvoll?

A Röteln-Antikörpertiter bei allen Frauen mit Kinderwunsch

B bei Immunsuppression

C nach schwerer Lokalreaktion

D nur vor notwendigen Impfungen in der Schwangerschaft

E vor Impfungen gegen die saisonale Influenza

4 Für welche Personengruppen empfiehlt die STIKO die Influenzaimpfung in Deutschland?

A nur für Personen ab dem 60. Geburtstag

B für Personen ab dem 50. Geburtstag und Personen mit chronischen Erkrankungen

C für Personen ab dem 60. Geburtstag, mit chronischen Erkrankungen, Schwangere und Personen mit umfangreichem Publikumsverkehr sowie Reisende

D Kinder bis zum 5. Lebensjahr und Personen ab dem 60. Geburtstag

E Personen ab dem 60. Geburtstag, mit chronischen Erkrankungen, mit umfangreichem Publikumsverkehr und Kinder bis zum 18. Geburtstag

5 Welche Impfungen sind zu unterlassen, wenn in der Umgebung eines Impflings Immundefiziente oder Schwangere sind?

A Alle Lebendimpfstoffe sind verboten.

B Nur die Gelbfieberimpfung ist ausgeschlossen.

C Keine Impfung ist ausgeschlossen.

D Varizellenimpfung und FSME-Impfung.

E Nur die Varizellenimpfung. 
Welche Aussage ist richtig? Eine stillende Wöchnerin hat keinen Schutz gegen Keuchhusten, Masern und Poliomyelitis.

Ich impfe sie...

A auf keinen Fall in der Stillzeit.

B mit Tdap-Impfstoff, MMR und IPV erst nach der Stillzeit.

C nur gegen Keuchhusten und Poliomyelitis, Lebendimpfstoffe sind in der Stillzeit verboten.

mit Tdap-IPV-Vierfachimpfstoff und mindestens mit 14 Tagen Abstand MMR.

mit Tdap-IPV-Vierfachimpfstoff und gleichzeitig mit dem Lebendimpfstoff MMR.

\section{Welche der folgenden Aussagen ist falsch?}

A Eine vor > 20 Jahren durchgeführte Grundimmunisierung gegen FSME braucht nicht wiederholt zu werden.

B Bei schriftlicher Aufklärung vor Impfung kann die mündliche Aufklärung entfallen.

C Alle Kontaktpersonen von Neugeborenen sollten vor Pertussis geschützt sein.

D Medizinische Fachangestellte dürfen impfen.

E Auch Jungen und Männer profitieren von einer HPV-Impfung.

8 Welche Aussage bzw. Empfehlung zur Senkung des Infektionsrisikos von Neugeborenen ist richtig?

A Trennung von Mutter und Kind bei Infektionsgefahr.

B Im ersten Lebensjahr besteht ein sicherer Nestschutz.

C Bereits während der Schwangerschaft alle Impflücken schließen.

D Alle Kontaktpersonen von Neugeborenen sollten geschützt sein vor Influenza, Masern, Pertussis.

E Keine Lebendimpfstoffe im Wochenbett impfen.

$9 \quad$ Was gilt als absolute Kontraindikation für eine Impfung?

A bevorstehende Operation

B Anfallsleiden oder Fieberkrämpfe in der Anamnese

C Allergien gegen Bestandteile des Impfstoffs

D Totimpfstoffe bei Chemo- oder anderer immunsupprimierender Therapie

E Multiple Sklerose und Neurodermitis

10 Eine Mutter kommt mit ihrer 9-jährigen Tochter in die Praxis und lässt sich über die HPV-Impfung aufklären. Der Impfausweis der Tochter enthält altersentsprechend alle nach STIKO erforderlichen Impfungen bis zum 5. Lebensjahr. Ich impfe im 2-Dosen-Schema den nonavalenten HPV-Impfstoff ...

A und gleichzeitig frische ich Hepatitis B, Tetanus, Diphtherie und Poliomyelitis auf.

B und gleichzeitig frische ich Tetanus, Diphtherie, Pertussis und Poliomyelitis auf, Hepatitis B-Auffrischung erst 4 Wochen später.

C Neben einer HPV-Impfung sind keine weiteren Impfungen zeitgleich erlaubt.

D nicht, weil das Mädchen noch so jung ist, dass keine Sexualkontakte zu erwarten sind. Ansonsten ist der Impfausweis komplett

E und gleichzeitig 1× Tdap-IPV.

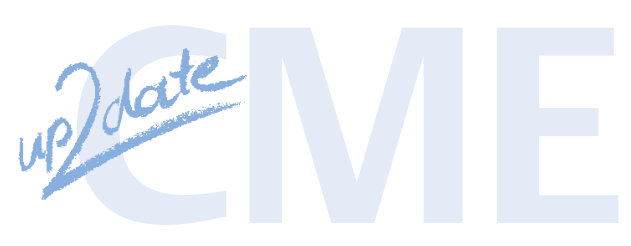

